\title{
The role of proton precipitation in the excitation of auroral FUV emissions
}

\author{
B. Hubert and J.-C. Gérard \\ Laboratoire de Physique Atmosphérique et Planétaire, Institut d'Astrophysique et de \\ Géophysique, Université de Liège, Liège, Belgium \\ D. V. Bisikalo and V. I. Shematovich \\ Institute of Astronomy, Russian Academy of Sciences, Moscow, Russia \\ S. C. Solomon \\ Laboratory for Atmospheric and Space Physics, University of Colorado, Boulder, Colorado
}

\begin{abstract}
Far ultraviolet remote sensing from a high-altitude satellite is extensively used to image the global aurora, derive its energetics, and follow its dynamical morphology. It is generally assumed that the observed emissions are dominated by the interaction of the precipitated electrons with the thermospheric constituents. A model to calculate far ultraviolet emissions excited by auroral electrons and protons and the secondary electrons they generate has been used to calculate the volume excitation rate of the H I Ly- $\alpha$, O I 1304 and $1356 \AA$, N I $1493 \AA$ multiplets, and the $\mathrm{N}_{2}$ Lyman-Birge-Hopfield (LBH) bands. The characteristic energy and the energy flux are derived from the observed statistical distribution of precipitated protons and electrons. This model is applied to the midnight aurora, the noon cusp, and a proton-dominated aurora for moderately disturbed conditions. We show that in the first two cases, direct electron impact dominates the vertically integrated emission rate over the proton component, although proton excitation plays an important role at some altitudes in the daytime cusp. In afternoon regions of the auroral zone near the auroral boundary, secondary electrons due to proton ionization are the main source of FUV emissions. The energy dependence of the efficiency of LBH band emission viewed from high altitude is calculated for electron and proton precipitations. Maps of the $\mathrm{N}_{2} \mathrm{LBH}$ emission excited by both components are obtained, and regions of proton-dominated auroral emission are identified. It is found that the distribution of the ratio of proton-induced to electron-induced brightness resembles maps of the ratio of the respective precipitated energy fluxes. Proton-dominated FUV emissions are thus located in a C-shaped sector extending from prenoon to midnight magnetic local times with a maximum proton contribution near the equatorward boundary of the statistical electron oval. The distribution of the Ly- $\alpha / \mathrm{LBH}$ intensity ratio is found to mimic the ratio of the proton flux/total energy flux, although it is insufficient by itself to accurately determine the relative fraction of auroral energy carried by the protons.
\end{abstract}

\section{Introduction}

Global observations from space in the far ultraviolet (FUV) make it possible to image the aurora in daytime and nighttime conditions. Remote sensing of the entire polar region from highly excentric orbits provides morphological observations, and time-dependent imaging of the FUV aurora provides key information on the dynamical processes occurring in various regions of the

Copyright 2001 by the American Geophysical Union.

Paper number 2000JA000288.

0148-0227/01/2000JA000288\$09.00 magnetosphere. The measured brightness of specific lines or molecular bands may be converted into a corresponding flux of charged particles into the atmosphere if the excitation mechanisms are identified. This conversion of apparent emission rates into physical flux units requires the knowledge of relevant excitation, absorption and scattering cross sections, and density profiles of the atmospheric constituents. This technique was succesfully applied to FUV cameras on board several satellites. The implicit assumption generally used to convert emission rates to incident energy fluxes is that electrons carry the bulk of the precipitated auroral energy flux [Ishimoto et al., 1988; Germany et al., 1997]. How- 
ever, ground-based observations [Eather, 1967; Lummerzheim and Galand, 2001] show the presence of the $\mathrm{H}_{\alpha}$ and $\mathrm{H}_{\beta}$ lines of the Balmer series of atomic hydrogen. These lines are unambiguous signatures of the presence of auroral protons. They are excited in charge exchange collisions of precipitated protons with atmospheric constituents. In these collisions the incoming fast proton captures an electron from a neutral particle $\left(\mathrm{N}_{2}, \mathrm{O}_{2}\right.$, or $\left.\mathrm{O}\right)$, producing an ion and a fast $\mathrm{H}$ atom in an excited state. During deexcitation, Doppler-shifted photons are emitted by the fast $\mathrm{H}$ atoms producing a broadened and shifted $\mathrm{H}$ I line. In situ satellite observations also indicate that protons are generally present in the particle beam interacting with the atmosphere [Hardy et al., 1989]. Doppler-shifted Lyman- $\alpha$ (Ly- $\alpha)$ emission has been spectrally observed from space [Ishimoto et al., 1988, 1989] and has been recently imaged [Mende et al., 2001] with the Spectrographic Imager on board the Imager for Magnetopause-to-Aurora Global Exploration (IMAGE) satellite [Mende et al., 2000].

Numerous in situ measurements of energetic protons suggest that protons may dominate the budget of energy deposited in the atmosphere at selected local times and latitudes. The statistical distribution of the precipitating auroral ions [Hardy et al., 1989] indicates that the maximum energy flux occurs in C-shaped regions symmetric about a meridian running prenoon to premidnight. For example, for $K p=5$ a maximum of 0,25 erg cm $\mathrm{cm}^{-2} \mathrm{~s}^{-1} \mathrm{sr}^{-1}$ is found in the premidnight sector. Globally, the protons carry $11-17 \%$ of the total energy precipitated into the atmosphere. The difference in shape with the corresponding statistical pattern of electron precipitation [Hardy et al., 1985] is such that the statistical proton energy flux may locally exceed the electron flux.

The FUV auroral spectrum is dominated by the $\mathrm{N}_{2}$ Lyman-Birge-Hopfield (LBH) bands, H I Ly- $\alpha$, the O I multiplets at 1304 and $1356 \AA$, and several N I lines [Crosswhite and Fastie, 1962; Gérard and Barth, 1976; Feldman and Gentieu, 1982; Ishimoto et al., 1988]. All transitions with the exception of $\mathrm{H}$ I Ly- $\alpha$ are excited by electron impact on $\mathrm{N}_{2}, \mathrm{O}_{2}$, and $\mathrm{O}$ in the electron aurora. In the proton aurora the emissions are produced by collisions of protons and fast $\mathrm{H}$ atoms with the atmospheric constituents as well as by secondary electrons produced during ionization by the $\mathrm{H}^{+}-\mathrm{H}$ beam.

Several methods have been used to investigate the interaction of proton and hydrogen auroral fluxes with the atmosphere and to calculate their optical signatures. These approaches can be generally categorized as (1) range method [Rees, 1982], (2) linear transport theory [Jasperse and Basu, 1982; Basu et al., 1987, 1993;Galand et al., 1997; Lummerzheim and Galand, 2001], and (3) Monte Carlo test particle method [Kozelov and Ivanov, 1992; Synnes et al., 1998; Lorentzen et al., 1998]. These numerical methods allow the evaluation of several effects induced by proton precipitation such as escape fluxes, heating rate of the atmospheric gas, and optical emission rates. A more detailed review of the various methods used to calculate electron and proton transport, their advantages, and their drawbacks is given by Solomon [2001]. Decker et al. [1996] compared results obtained by three different methods and found good agreement for calculated quantities. Excitation of FUV auroral emissions was discussed by Strickland et al. [1993], who calculated emission yields for the excitation of several FUV features by electrons and protons.

Recently, Gérard et al. [2000] showed that it is important to take into account the stochastic nature of collisional scattering to properly describe the behavior of high-energy protons (or $\mathrm{H}$ atoms) which collide with the target particles and change their direction following a probabilistic distribution of the scattering angle. This feature is an important component of the kinetic model used in this work to calculate the proton transport. A second advantage of this model was the use of updated cross sections and scattering angles based on experimental data.

In this study we combine an electron energy degradation code with a proton Monte Carlo model to calculate the relative contribution of individual processes to the excitation of the main FUV auroral features in the presence or not of solar-induced emissions. We discuss the importance of the protons and their FUV signatures for different typical auroral conditions. Regions where protons make a significant contribution to the total FUV emission rates are identified on the basis of the statistical morphology derived from in situ particle measurements. We discuss whether the Ly- $\alpha / \mathrm{LBH}$ intensity ratio may be used to remotely sense the relative energy flux carried by auroral protons relative to electrons.

\section{The Model}

The methodology adopted to calculate the excitation rates of the FUV emissions rests on the combination of transport models describing the interaction of an electron and a proton beam, respectively, with the atmosphere.

The calculation of auroral electron energy degradation and excitation by solar-induced processes is based on the GLOW model [Solomon et al., 1988; Solomon, 2001] with updated cross sections. An initial energy distribution of the primary electrons is prescribed in an analytical form. The two-stream transport algorithm is based on Banks et al. 's [1974] code, extended to higher energies for auroral calculations. The local electron flux is calculated using the two-stream approximation, solving for the upward and downward hemispherical fluxes along a magnetic field line assumed to be vertical as a function of energy. Elastic collisions, discrete energy losses from inelastic collisions, and heating of the thermal electrons are considered. The energy bins and the altitude steps are variable in size. The inelastic cross sections are used in the analytical form given by Jackman et al. [1977] and Green and Stolarski [1972]. Tests have shown that energy conservation is ensured with an accuracy better than $10 \%$. Many of the excitation cross sections were refit to reflect subsequent revisions, as described by Solomon et al. [1988]. Monte Carlo and multistream methods provide a description of the 
Table 1. Electron and Proton Émission Cross Sections ${ }^{a}$

\begin{tabular}{llcc}
\hline Feature & Process & Peak value, $10^{-17} \mathrm{~cm}^{2}$ & Peak Energy, eV \\
\hline $\mathrm{O}$ I 1304 $\AA$ & $e+\mathrm{O}$ & 2.4 & 23 \\
& $e+\mathrm{O}_{2}$ & 0.32 & 80 \\
& $\mathrm{H}+\mathrm{O}$ & 0.29 & 3730 \\
& $\mathrm{H}+\mathrm{O}_{2}$ & 0.41 & 3730 \\
$\mathrm{O}$ I $1356 \AA$ & $e+\mathrm{O}$ & 1.1 & 16 \\
& $e+\mathrm{O}_{2}$ & 0.88 & 110 \\
& $\mathrm{H}+\mathrm{O}$ & 1.0 & 1650 \\
& $\mathrm{H}+\mathrm{O}_{2}$ & 1.4 & 1650 \\
$\mathrm{~N}_{2}$ LBH & $e+\mathrm{N}_{2}$ & 3.0 & 20 \\
& $\mathrm{H}^{+}+\mathrm{N}_{2}$ & 6.0 & 16 \\
& $\mathrm{H}+\mathrm{N}_{2}$ & 4.7 & 16 \\
$\mathrm{~N}$ I 1493 $\AA$ & $e+\mathrm{N}_{2}$ & 0.18 & 85 \\
$\mathrm{H} \mathrm{I} \mathrm{Ly-} \alpha$ & $\mathrm{H}^{+}+M$ & & \\
& $\mathrm{H}+M$ & & \\
\hline${ }^{a}$ For the peak value of $\mathrm{H}$ I Ly- $\alpha$, please see the text.
\end{tabular}

angular distribution of the energetic particles which is missing in a two-stream approach. However, in a recent comparative study, Solomon [2001] showed that the vertical ionization profiles calculated with the twostream method implemented in the GLOW model are in excellent agreement with those obtained with more sophisticated Monte Carlo and multistream methods. For its simplicity of use and reduced computing time, we adopt the simpler two-stream approach.

The proton energy transport code was described in detail by Gérard et al. [2000]. It is based on the direct Monte Carlo method [Marov et al., 1997], which is a stochastic implementation of the solution of the Boltzmann equations for the $\mathrm{H}^{+}-\mathrm{H}$ beam. The model was validated against other model results using different approaches but the same proton flux characteristics [Decker et al., 1996]. We note that the energy flux used as an input of the Monte Carlo model for the case illustrated in Figure 2 of Gérard et al. [2000] was actually 0.3 instead of $0.5 \mathrm{erg} \mathrm{cm}^{-2} \mathrm{~s}^{-1}$. Therefore the peak of the energy deposition for the Monte Carlo simulation must be scaled up by a factor of 1.7 , bringing the two curves into closer agreement.

Once the protons reach the thermosphere, charge exchange collisions with ambient $\mathrm{O}, \mathrm{O}_{2}$, and $\mathrm{N}_{2}$ generate a population of fast $\mathrm{H}$ atoms which, in turn, may be converted back to energetic protons. The Monte Carlo proton aurora code includes a detailed calculation of all elastic and inelastic processes for both species. A detailed treatment of the momentum transfer in all collisions makes it possible to follow the pitch angle distribution of the simulated particles. Three sources of velocity vector redistribution of the energetic protons are considered: (1) magnetic mirroring in the dipole geomagnetic field, (2) geometric spreading caused by the convergent magnetic field lines, and (3) collisional angular redistribution. Doubly differential collision cross sections are used to describe proton and $\mathrm{H}$ atoms' collisions from 0.5 to $5 \mathrm{keV}$, with extrapolations at higher energies. Ionization of the major constituents by collisions with energetic $\mathrm{H}^{+}$and $\mathrm{H}$ particles generates secondary electrons:

$$
\mathrm{H}_{f}^{+}+\mathrm{M} \rightarrow \mathrm{H}_{f}^{+}+\mathrm{M}^{+}+e,
$$

$$
\begin{gathered}
\mathrm{H}_{f}+\mathrm{M} \rightarrow \mathrm{H}_{f}+\mathrm{M}^{+}+e, \\
\mathrm{H}_{f}+\mathrm{M} \rightarrow \mathrm{H}_{f}^{+}+\mathrm{M}+e,
\end{gathered}
$$

where subscript $f$ denotes a fast particle and $M$ is one of the major constituents.

The energy production rate of secondary electrons by these processes is calculated on the basis of the ionization and electron stripping cross sections by Edgar et al. $[1973,1975]$. The energy distribution of the ejected electron population is given by Rudd [1979] and Basu et al. [1993].

To simulate a combined electron-proton aurora this population of proton-generated secondary electrons is added to the electron-generated component. Their energy loss is calculated similarly to the energetic electron component. The temperature profile and the $\mathrm{O}, \mathrm{O}_{2}$, and $\mathrm{N}_{2}$ densities are calculated from the Mass Spectrometer Incoherent Scatter-1990 (MSIS-90) model atmosphere [Hedin, 1991]. Ionosphere electron temperature and density are taken from the International Reference Atmosphere-1990 (IRI-90) model [Bilitza, 1990].

The input energy flux and characteristic mean energy of both types of charged particles for prescribed geophysical conditions and geographic location are provided by the statistical precipitation model described by Hardy et al. [1987, 1991]. The resulting volume excitation rates for $L y-\alpha, N$ I $1493 \AA$, O I 1304 and 1356 $\AA$, and $\mathrm{N}_{2}$ LBH bands are calculated including all photo excitation and collisional excitation processes. Finally, the emerging intensities for a given view angle are calculated taking absorption into account.

The cross sections adopted to calculate the FUV emission rates are listed in Table 1 . The O I $1304 \AA$ electron impact excitation cross section is that of Zipf and Erdman [1985]. The O I $1356 \AA$ cross section was obtained by reducing the measured value of Stone and Zipf [1974] by a factor of 2.8, in agreement with the recommendation by Zipf and Erdman [1985]. The total $\mathrm{N}_{2}$ LBH cross section is taken from Ajello and Sheman$s k y$ [1985]. The absolute cross section of the $\mathrm{N} \mathrm{I}{ }^{2} \mathrm{D}-$ ${ }^{2} \mathrm{P}$ line at $1493.3 \AA$ was based on the measurements by Ajello and Shemansky [1985] at $100 \mathrm{eV}$. The shape was assumed to follow that given by the measurements 
of Mumma and Zipf [1971]. Many of the proton excitation cross sections for FUV emission are still poorly known or undetermined. The cross section leading to the production of the $\mathrm{H}(2 s)$ state by collisional excitation of fast $\mathrm{H}$ atoms and charge exchange with $\mathrm{O} . \mathrm{O}_{2}$, and $\mathrm{N}_{2}$ as described in detail by Gérard et ai. $[2000]$. The Ly- $\alpha$ excitation cross sections for collisions of fast $\mathrm{H}^{+}$and $\mathrm{H}$ with $\mathrm{O}_{2}$ and $\mathrm{N}_{2}$ are from Van $\mathrm{Zyl}$ and $\mathrm{Neu}-$ $\operatorname{mann}[1988]$. The value for impact on $\mathrm{O}$ was set equal to 0.7 times the $\mathrm{O}_{2}$ cross section. The excitation cross section of the O I $3 \mathrm{~s}^{3} S$ state by fast $\mathrm{H}$ atoms is taken from Edgar et al. [1975], and we assume again a value of 0.7 that of dissociative excitation of $\mathrm{O}_{2}$. As indicated by Strickland et al. [1993], the $1356 \AA$ emission cross section by $\mathrm{H}^{+}$is negligible owing to the nature of the transition. The value for collisions of $\mathrm{H}$ with $\mathrm{O}$ and $\mathrm{O}_{2}$ are adopted from Edgar et al. [1975]. As will be seen in section 3.2., these contributions are less than those of the secondary electrons, so that the exact value of the cross sections is not essential in this study. The LBH emission cross section due to $\mathrm{H}^{+}$and $\mathrm{H}$ collisions is from Kozelov and Ivanov [1992]. Because of the lack of reliable laboratory data, direct excitation of the 1493 $\AA$ emission by protons and $\mathrm{H}$ atoms is not considered.

The average energy and the total energy flux carried by auroral electron and protons as a function of location, time, and geophysical conditions are given by Hardy et al.'s [1987, 1991] empirical models based on data collected over several years with detectors on board the Defense Meteorological Satellite Program (DMSP) satellites. The measured vertical energy flux (in eV $\mathrm{cm}^{-2} \mathrm{~s}^{-1} \mathrm{sr}^{-1}$ ) is converted into a total energy flux assuming that the precipitation of both types of particles is isotropic over one hemisphere. Since the high-energy cutoff of the detectors is $30 \mathrm{keV}$, Hardy et al. [1987, 1991] estimated the high-energy component up to 100 $\mathrm{keV}$ using a simple extrapolation procedure. They derived the mean particle energy from the energy spectrum above $30 \mathrm{eV}$. Robinson et al. [1987] pointed out that owing to the excess incident electron flux from a Maxwellian at energies below $500 \mathrm{eV}$, the mean energy provided may be lower than a Maxwellian fit to the data. As an approximation, we assume a Maxwellian distribution with characteristic energy $E_{0}$ for electrons such that the mean energy $\left(\bar{E}=2 E_{0}\right)$ is equal to the mean electron energy determined from Hardy et al.'s extrapolated energy spectra. An estimate of the error resulting from the use of a single Maxwellian distribution over the full range of electron energies may be obtained from the discussion of the electron spectral shape measured with the DMSP detectors by Hardy et al. [1985]. They calculated the average energy and energy flux separately for electrons with $50 \mathrm{eV} \leq E<660 \mathrm{eV}$ and $E>660 \mathrm{eV}$ as a function of $K p$. On the basis of these numbers, we determine the contributions of both populations to the absorbed LBH emission rate. It is found that for a total $1 \mathrm{erg} \mathrm{cm} \mathrm{cm}^{-2} \mathrm{~s}^{-1}$ precipitation, the soft component $(<660 \mathrm{eV})$ contributes $0.08 \mathrm{kR}$, and the higher-energy flux contributes $0.81 \mathrm{kR}$, for a total of $0.89 \mathrm{kR} / \mathrm{erg} \mathrm{cm}^{-2} \mathrm{~s}^{-1}$. If the energy distribution is approached by a Maxwellian with the same mean energy; the calculated LBH emission is $0.95 \mathrm{kR}$. The error due to the use of a single Maxwellian instead of the sum of two Maxwellian distributions in this case is therefore $6 \%$ but may reach up to $30 \%$ for the O I $1356 \AA$ emission.

For protons, measurements suggest that a kappa distribution provides a better fit. Lyons and Evans [1984] showed that the proton distributions at ionospheric altitudes have high-energy components. Sharber et al. [1993] also observed with the UARS satellite ion spectra with high-energy tails similar to those measured by Christon et al. [1991] in the central plasma sheet. Consequently, we determine the $E_{0}$ and $\kappa$ parameters fitting in such a way that the mean energy $\bar{E}=2 E_{0}(\kappa /(\kappa-2))$ is equal to Hardy et al. [1991] value. The $\kappa$ index was determined by fitting the average proton energy spectra given by Hardy et al.'s [1989] Figure 7 for $K p=3$ at four different local times. As for incident protons, Hardy et al. [1989, 1991] derived the total energy flux by extrapolating the particle flux toward high energies by a Maxwellian. We use a kappa law, which decreases the relative importance of the low-energy protons and increases that of the high-energy protons. This assumption places the emission peak at a lower altitude than it would with a Maxwellian, causing additional absorption by $\mathrm{O}_{2}$. The difference between the two spectral contributions was assessed by comparing the outputs from two runs: (1) a kappa distribution characterized by $\bar{E}=9.3 \mathrm{keV}$ and $\phi_{0}=1 \mathrm{erg} \mathrm{cm}{ }^{-2} \mathrm{~s}^{-1}$ and (2) a Maxwellian distribution with the same $\bar{E}$ and $\phi_{0}$. The results indicate a $19 \%$ smaller vertical brightness for the LBH intensity and a $17 \%$ smaller value for $L y-\alpha$ in the case of the kappa distribution.

All photodissociation, photoionization, photoelectron, and excitation processes in the dayglow are calculated by the GLOW model. The $1304 \AA$ triplet is excited by resonance scattering in addition to electron impact on $\mathrm{O}$ and $\mathrm{O}_{2}$. The $1356 \AA$ doublet is solely produced by photoelectron impact on $\mathrm{O}$ and $\mathrm{O}_{2}$. The $\mathrm{N}_{2} \mathrm{LBH}$ bands and the N I lines are produced by photoelectron impact on ground state $\mathrm{N}_{2}$. Doppler-shifted hydrogen emission is not excited in the dayglow.

The volume emission rates can be integrated for an arbitrary view angle to simulate observations by a downward looking FUV instrument on board a high-altitude spacecraft. This integration is straightforward in optically thin cases such as Doppler-shifted Ly- $\alpha$ and N I $1493 \AA$ lines. In this case only attenuation by $\mathrm{O}_{2}$ absorption in the lower thermosphere must be considered. We use the $\mathrm{O}_{2}$ absorption cross section by Ogawa and Ogawa [1975]. The (6-0) band of LBH was shown by Conway [1983] to be optically thick at low altitudes. The total contribution of this band amounts to less than $10 \%$ of the total system intensity. In addition, absorption by $\mathrm{O}_{2}$ becomes important in the region where optical depth effects in this band become significant. We thus ignore multiple scattering for the $\mathrm{N}_{2} \mathrm{LBH}$ bands, but each line is attenuated by $\mathrm{O}_{2}$ and summed up to provide the total emergent LBH emission rate. Generally, attenuation may be important for emissions below $\sim 1550 \AA$ but becomes negligible at longer wavelengths for characteristic electron energies (less than 100 


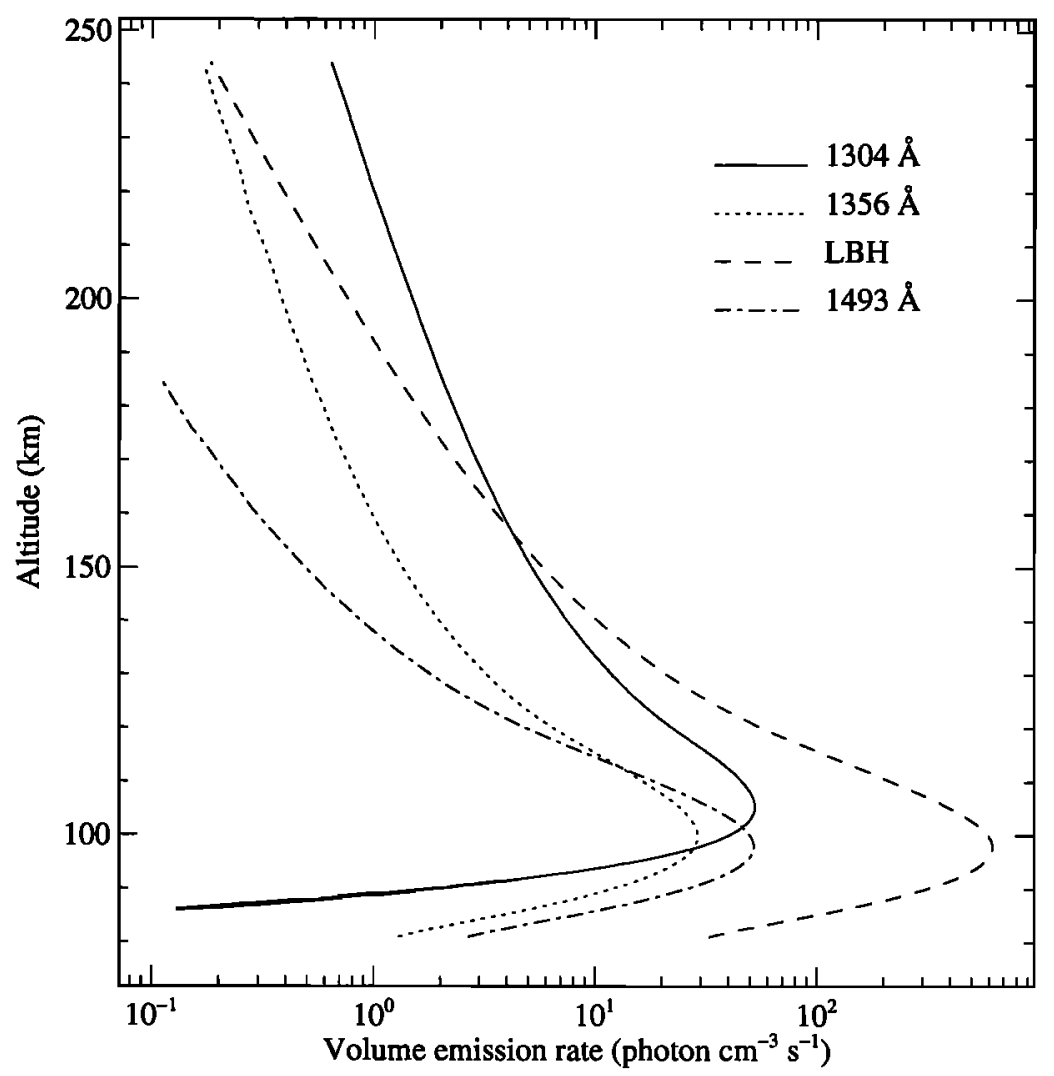

Figure 1. Vertical distribution of FUV excitation rates for a $1 \mathrm{erg} \mathrm{cm}^{-2} \mathrm{~s}^{-1}$ pure electron aurora for an initial Maxwellian energy distribution with $\bar{E}=20 \mathrm{keV}$.

$\mathrm{keV}$ ) typically encountered in the statistical database of Hardy et al. $[1987,1991]$ which locate the emission peak above $100 \mathrm{~km}$. Similarly, the two doublet components of the $1356 \AA$ iransition may be affected by multiple scattering. However, the efficiency is relatively small [Strickland and Anderson, 1983], and this effect is negligible for view angles close to zenith and emission peaks above $110 \mathrm{~km}$. It has been neglected in this work. We now analyze the relative role played by proton precipitation in producing FUV auroral emissions.

\section{Results}

\subsection{Pure Electron Aurora}

We first illustrate some model results obtained for a pure electron aurora. The solar and geomagnetic conditions correspond to nighttime high latitudes for maximum solar activity: December $25,65^{\circ} \mathrm{N}, 0000 \mathrm{LT}$, $F_{10.7}=F_{10.7 A}=191$. The local time and latitude are such that there is no contamination by dayglow. As an example, the volume emission rates of the O I 1304 $\AA, 1356 \AA$, N I $1493 \AA$, and LBH emissions are calculated for an isotropic electron precipitation having a Maxwellian energy distribution characterized by $\bar{E}=20$ $\mathrm{keV}$ and a $1 \mathrm{erg} \mathrm{cm}^{-2} \mathrm{~s}^{-1}$ energy flux (Figure 1). This mean energy is typical of electron energies encountered during substorms in the midnight sector of the auroral oval. The $\mathrm{N}_{2} \mathrm{LBH}$ is the dominant emission of the
FUV spectrum, showing a peak at $99 \mathrm{~km}$. The 1304 $\AA$ emission peaks slightly higher than both the $1356 \AA$ and the LBH bands. The $1356 \AA$ emission has a larger low-altitude contribution than $1304 \AA$ does, owing to the larger contribution of dissociative excitation of $\mathrm{O}_{2}$. Multiple scattering of the $1304 \AA$ triplet is important but was not considered here. Radiative transfer calculations have shown that the fraction of photon backscattered upward varies with the altitude of the emission peak in a complex way. When the mean electron energy increases, the altitude of the emission peak moves down into a region richer in $\mathrm{O}_{2}$, causing more absorption of the short-wavelength emissions. Therefore, as the emission peak reaches the region of $\mathrm{O}_{2}$ absorption, the probability that photons initially emitted downward are backscattered decreases from unity to zero. In the case shown here the attenuation by $\mathrm{O}_{2}$ is $11 \%$ for 1304 $\AA, 73 \%$ for $1356 \AA$, and $69 \%$ for the total LBH system. The calculated emerging intensity of the total LBH bands is $3.2 \mathrm{kR}$. This value is to be compared with Ishimoto et al. 's [1988] satellite nadir observation that the average emergent intensity of the unabsorbed $(3,10) \mathrm{LBH}$ band at $1928 \AA$ is $55 \mathrm{R} /$ incident erg $\mathrm{cm}^{-2}$ $\mathrm{s}^{-1}$ in $K p=3$ to 5 conditions. Since the (3-10) band accounts for $1.7 \%$ of the total LBH brightness, their total unabsorbed LBH emission rate is $3.3 \mathrm{kR}$, in excellent agreement with the case described here, although the exact value of $\bar{E}$ was not measured during these observations. 
Table 2. Geophysical Parameters of the Simulated Cases

\begin{tabular}{lccc}
\hline Parameter & Midnight & Daytime Cusp & Proton Aurora \\
\hline Day & Aug. 24 & Aug. 24 & Dec. 25 \\
Latitude & $66^{\circ} \mathrm{N}$ & $76^{\circ} \mathrm{N}$ & $63^{\circ} \mathrm{N}$ \\
Longitude & $0^{\circ}$ & $0^{\circ}$ & $0^{\circ}$ \\
Solar zenith angle & $103.4^{\circ}$ & $65.7^{\circ}$ & $98.4^{\circ}$ \\
MLT, & 0000 & 1200 & 1630 \\
$F 10.7$ & 191 & 191 & 191 \\
$F 10.7 A$ & 216 & 216 & 216 \\
$K p$ & 3 & 3 & 4 \\
$Q_{0}$ (electrons), erg cm & -2 & 0.01 \\
$Q_{0}$ (protons), $\mathrm{erg} \mathrm{cm}^{-2} \mathrm{~s}^{-1}$ & 2.7 & 0.26 & 0.15 \\
$\bar{E}$ (electrons), keV & 0.46 & 0.07 & 0.84 \\
$\bar{E}$ (protons), $\mathrm{keV}$ & 2.8 & 0.14 & 39.2 \\
$\kappa$ (protons) & 23.9 & 1.9 & 3.5 \\
\hline
\end{tabular}

\subsection{Combined Electron-Proton Aurora}

Both electron and proton precipitations are generally simultaneously present in the auroral region. To illustrate and assess the role of proton precipation, we use three different auroral cases corresponding to (1) a midnight electron-dominated aurora, (2) a noon cusp aurora characterized by a softer precipitation, and (3) a proton-dominated afternoon precipitation. Their geographic, geophysical, and solar activity conditions are listed in Table 2. All three correspond to moderately disturbed conditions $(K p=3)$.

3.2.1. Nightside aurora. The first case is nighttime aurora in the midnight sector (Magnetic Local Time $(M L T)=0000$, latitude $=66^{\circ}$ ) for solar maximum conditions. The mean proton energy is nearly 9 times as high as the electron energy, but the energy flux carried by protons is $\sim 15 \%$ of the total. This case was selected by taking the latitude giving the highest total energy flux precipitated in the atmosphere at $0000 \mathrm{MLT}$. The vertical distribution of the excitation rates for the $\mathrm{LBH}$ bands and the O I $1356 \AA$ is shown in Figures $2 a$ and $2 b$. The LBH excitation peaks at $115 \mathrm{~km}$ and is dominated by the electron component. Since the excitation cross section peaks near $20 \mathrm{eV}$, secondary electrons are more efficient than are primary electrons. The second source is the impact of secondary electrons due to ion- izations by the $\mathrm{H}^{+}-\mathrm{H}$ beam, which contributes $10 \%$. Finally, direct excitation by fast $\mathrm{H}^{+}$and $\mathrm{H}$ makes a $2 \%$ contribution to the total. The conclusion is similar for $1356 \AA$, which is also dominated by the (primary and secondary) electron component. However, in this case, direct $\mathrm{H}^{+}-\mathrm{H}$ impact and secondary electrons due to protons both contribute $\sim 12 \%$ at $135 \mathrm{~km}$, reaching their maximum contribution $\sim 20 \mathrm{~km}$ above the direct electron component.

Table 3 gives the vertically integrated emission rates of Ly- $\alpha, \mathrm{LBH}, 1304 \AA, 1356 \AA$, and $1493 \AA$ due to electron and protons separately. About $20 \%$ of the emerging LBH intensity is due to the proton component. The other proton contributions are $100 \%$ for $L y-\alpha, 17 \%$ for $1304 \AA$ and $20 \%$ for $1356 \AA$. Consequently, the proton contribution to the $\mathrm{O} I$ and $\mathrm{N}_{2} \mathrm{LBH}$ emissions is close to the fractional energy flux carried by protons in the precipitation (15\%).

3.2.2. Noon cusp aurora. Noon cusp conditions $\left(\mathrm{MLT}=1200\right.$, latitude $\left.=75^{\circ} \mathrm{N}\right)$ have been selected for the next case. The mean proton energy is now more than 10 times that of the electrons, and the proton energy flux is 0.26 of the electron energy flux. It corresponds to the latitude of maximum energy flux statistically precipitated in the noon cusp sector.

Figures $3 \mathrm{a}$ and $3 \mathrm{~b}$ show the vertical distribution of the LBH and $1356 \AA$ excitation rates. The LBH excita-

Table 3. FUV Vertically Integrated Emission Rates ${ }^{a}$

\begin{tabular}{lccccc}
\hline & Ly- $\alpha$ & LBH & $1304 \AA$ & $1356 \AA$ & $1493 \AA$ \\
\hline Midnight aurora & & & & & \\
Electrons & - & 2.9 & 1.5 & 0.39 & 0.23 \\
Protons & 1.1 & 0.8 & 0.31 & 0.096 & 0.02 \\
& & & & & \\
Noon cusp & - & 0.2 & 0.63 & 0.16 & 0.014 \\
$\quad$ Electrons & 0.6 & 0.05 & 0.023 & 0.01 & 0.001 \\
Protons & & & & & \\
& & & & & \\
Proton aurora & - & 0.001 & 0.02 & 0.005 & 0.001 \\
$\quad$ Electrons & 0.27 & 0.18 & 0.01 & 0.03 & 0.02 \\
\hline Protons & & & & &
\end{tabular}

${ }^{a}$ Units are kilorayleighs. 

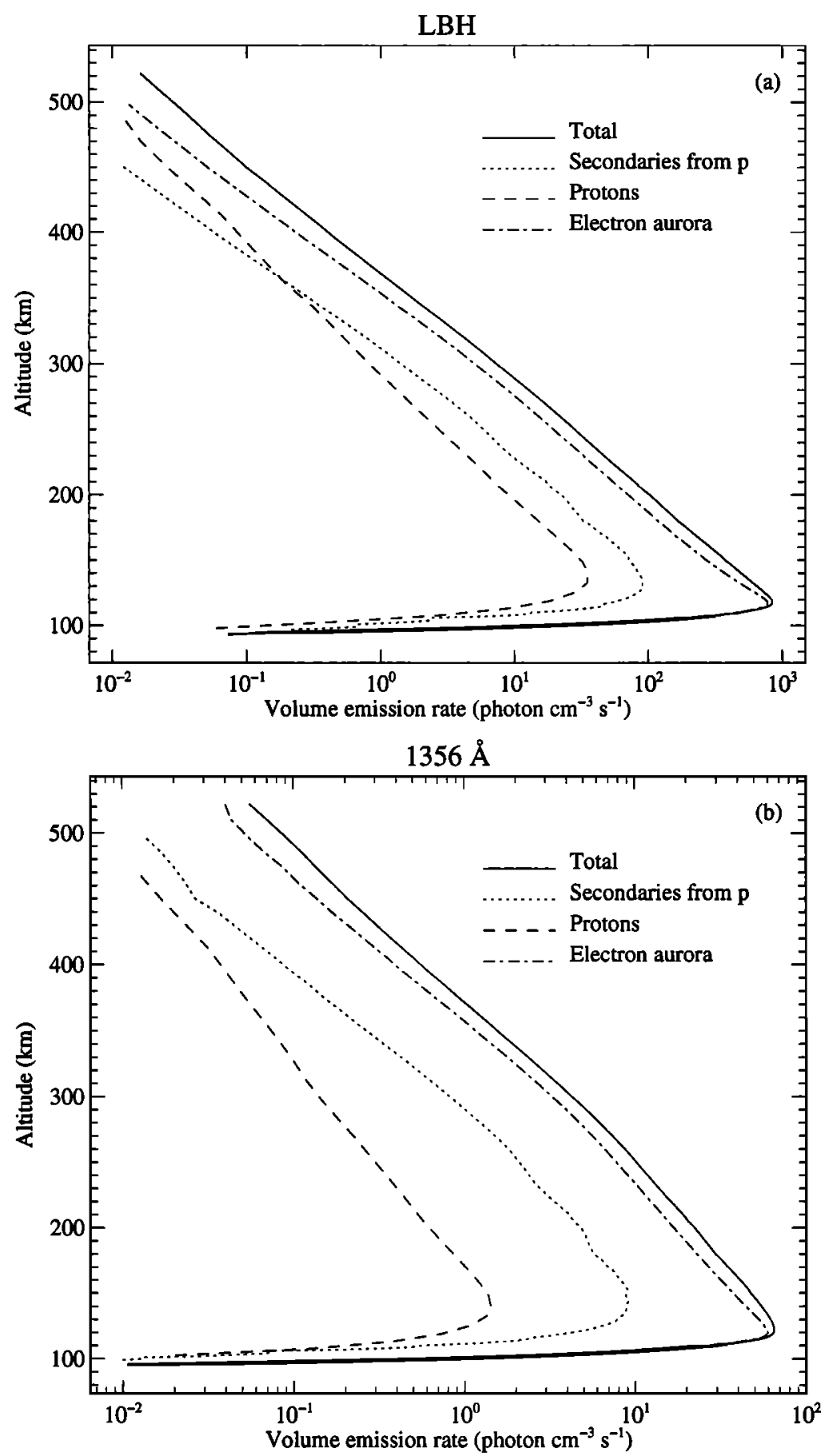

Figure 2. Vertical distribution of (a) the $\mathrm{N}_{2} \mathrm{LBH}$ and (b) O I $1356 \AA$ excitation rates for a midnight aurora.

tion is also largely dominated by the solar component (Figure 3a). The electron component (primaries and their secondaries) peaks at $280 \mathrm{~km}$, an altitude considerably higher than that in the previous case. This is a consequence of the low energy of the electrons associated with the noon polar cusp region. The direct $\mathrm{H}^{+}$ - $\mathrm{H}$ and their associated secondary electrons peak at lower altitude $(\sim 160 \mathrm{~km})$ and are both comparable in magnitude. The total production rate below $300 \mathrm{~km}$ is nearly totally solar-controlled with a small contribution from nonairglow processes. One should note, however, that the relative importance of the excitation by auroral particles compared to the solar (airglow) component will be variable under different conditions. In particular, it will vary with solar cycle and geomagnetic activity and with season through the solar zenith angle. The actual instantaneous energy flux may thus exceed the statistical value adopted here. The statistical auroral components may be viewed as statistically fixed values while the airglow contribution varies depending on sea- 

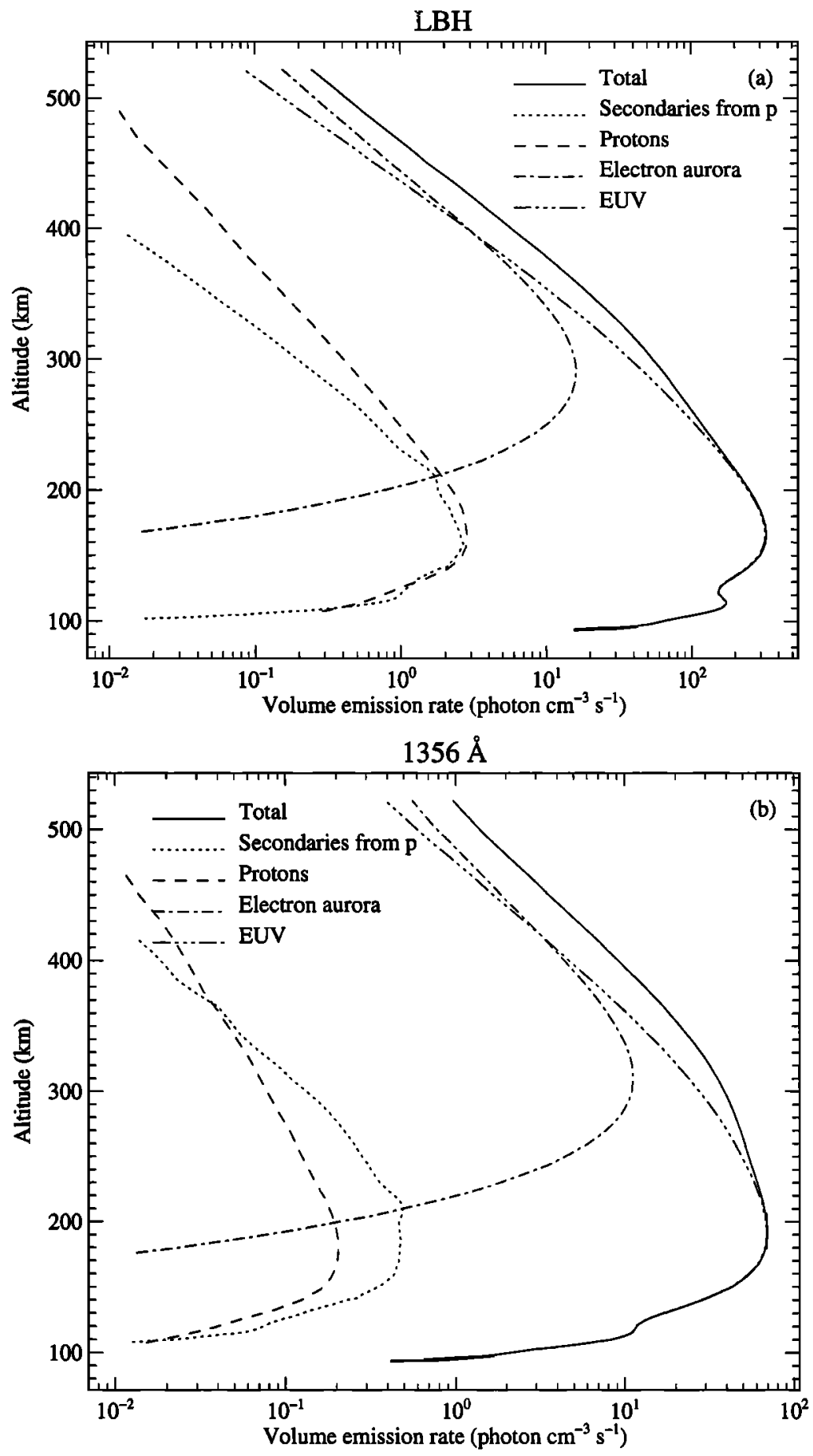

Figure 3. Vertical distribution of (a) the $\mathrm{N}_{2} \mathrm{LBH}$ and (b) $\mathrm{O}$ I $1356 \AA$ excitation rates for a noon cusp aurora

sonal conditions. The noon cusp aurora modeled here has two regimes with the low-altitude component dominated by protons and a second higher-altitude peak due to the electron component.

The $1356 \AA$ excitation (Figure $3 \mathrm{~b}$ ) has similar characteristics: Globally dominated by the airglow component, it also shows an upper region dominated by electrons and a lower-altitude region below $200 \mathrm{~km}$ mostly controlled by protons and their associated secondary electrons. The vertically integrated emission rates (Ta- ble 3) clearly confirm the minor role played by protons in comparison to electrons.

The two cases described so far correspond to typical auroral conditions $(K p=3)$ where the electron energy flux exceeds the proton component. We note that even in these conditions, the $\mathrm{H}^{+}-\mathrm{H}$ beam interaction with the atmosphere can generate altitude regions where excitation of the FUV emission is primarily controlled by protons in the lower part of the thermosphere under nonsunlit conditions. 
3.2.3. Proton-dominated aurora. We now examine conditions where the statistical proton precipitation accounts for most of the local auroral energy flux in the Northern Hemisphere. These conditions are observed in the afternoon sector near the equatorward boundary of the statistical precipitation oval. Considering all levels of geomagnetic activities, the ratio $Q_{0, p} / Q_{0, e}$ of the precipitated proton to the electron energy flux reaches a maximum value of 14 near 1600 MLT and $63^{\circ} \mathrm{N}$ for $K^{\prime} p=4$ (Table 2). The proton flux is $\sim 2$ times as high as that in the noon cusp but is only 0.32 of the midnight auroral case. The electron flux in this sector is very weak: 0.04 times its cusp value and 0.004 times the midnight aurora. It may thus be considered as a case of quasi-pure proton aurora in the premidnight sector such as those described on the basis of Balmer line observations by Vallances-Jones [1974], Mende and Eather [1976], and Creutzberg et al. [1988]. The estimated mean proton energy ( $39 \mathrm{keV}$ ) is higher than both in the cusp and midnight cases and exceeds the energy range of the satellite detectors. It results from the extrapolation procedure described by Hardy et al. [1989] and must be considered as indicative only. Figures $4 a-$ $4 \mathrm{e}$ show the volume excitation rates of the LBH, $1356 \AA$,
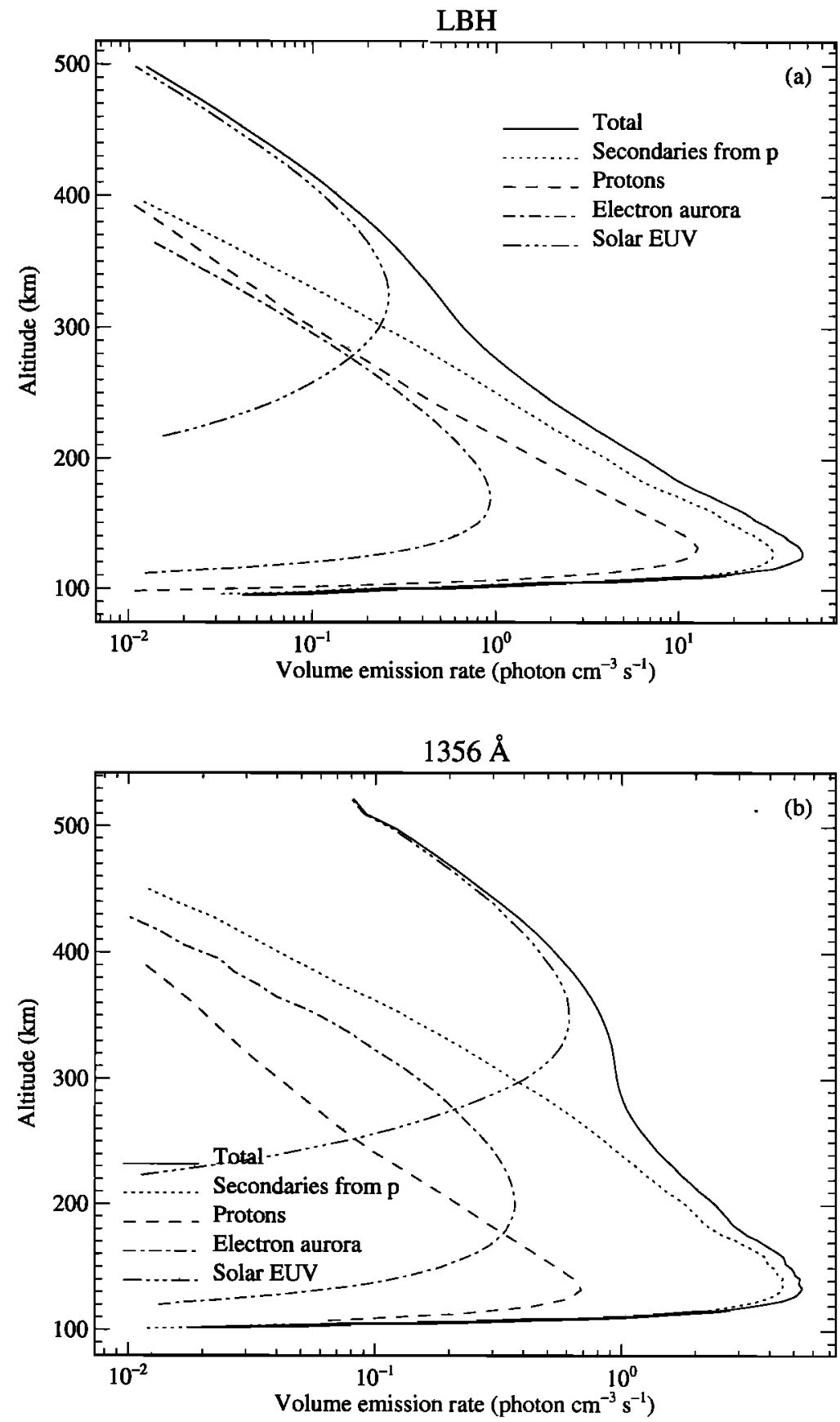

Figure 4. Vertical distribution of the excitation rate for proton-dominated aurora. (a) LBH bands. (b) O I $1356 \AA$; (c) O I $1304 \AA$, (d) N I $1493 \AA$ and (e) Ly- $\alpha$. 

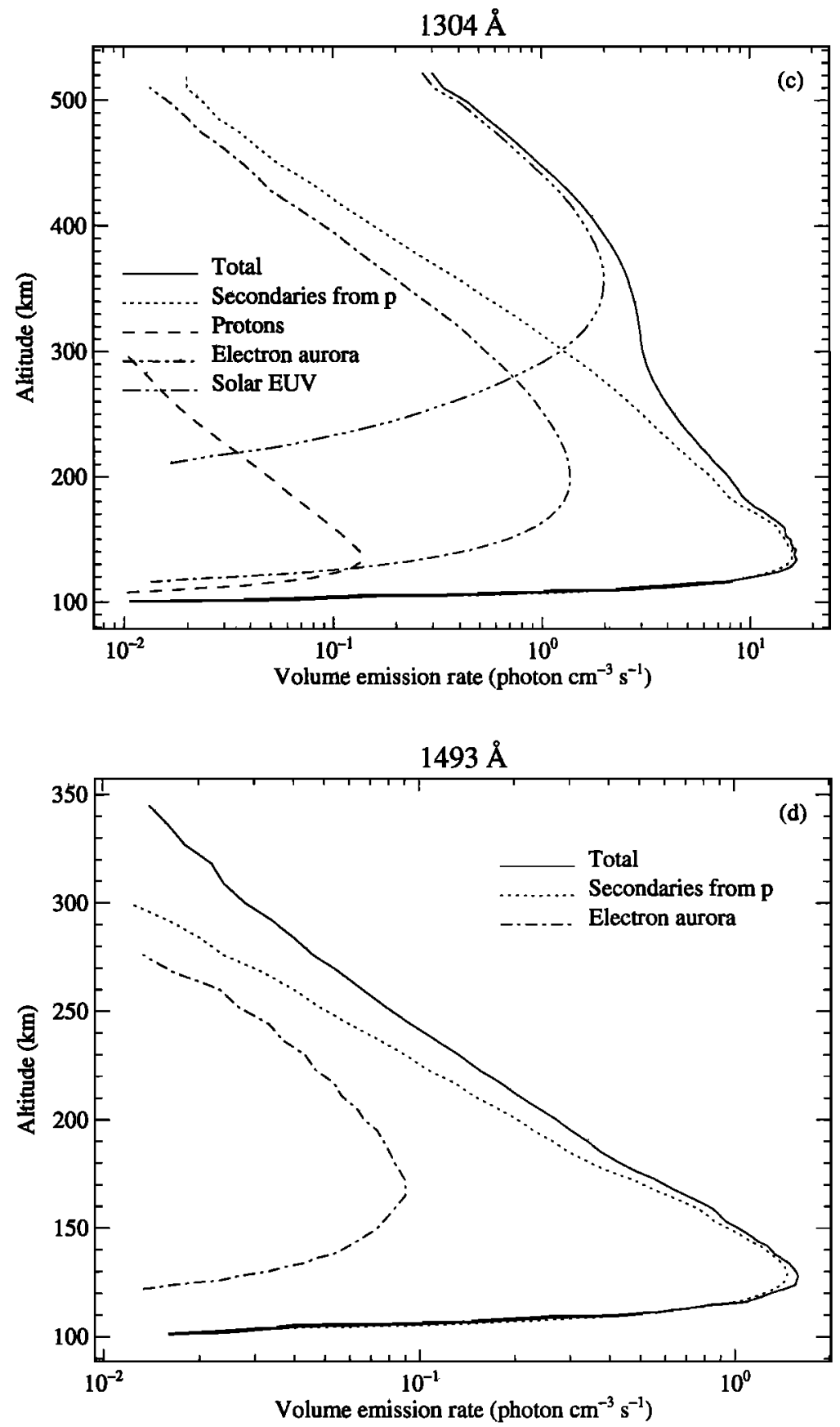

Figure 4. (continued)

$1304 \AA$, and $1493 \AA$ emissions, respectively, calculated for winter solstice conditions. The LBH band excitation (Figure $4 a$ ) is dominated by secondary electrons due to proton impact. The second most important source is direct $\mathrm{H}^{+}-\mathrm{H}$ excitation, which contributes $20 \%$ to the LBH band excitation at the peak. Excitation by primary electrons and their secondaries is a minor source of emission at all altitudes. Because of the large value of the solar zenith angle, the dayglow component is only significant above $\sim 300 \mathrm{~km}$ at solstice and contributes $1 \%$ to the total column.

The situation is qualitatively similar for O I 1356 $\AA$ (Figure $4 \mathrm{~b}$ ), where the proton-induced component is dominant. However, direct impact by protons and $\mathrm{H}$ atoms plays a relatively less important role than it does for the LBH bands. The direct electron contribution peaks near $200 \mathrm{~km}$, reflecting the low mean energy of the auroral electrons precipitated in this region. The dayglow component is negligible below $300 \mathrm{~km}$, although once integrated over the vertical column, it also contributes $\sim 16 \%$ to the total emission.

The $1304 \AA$ triplet (Figure 4c) is primarily excited by secondary electrons from proton impact, and direct proton excitation plays a very minor role. This is a consequence of the shapes of the secondary electron spectrum and the electron excitation cross section. 


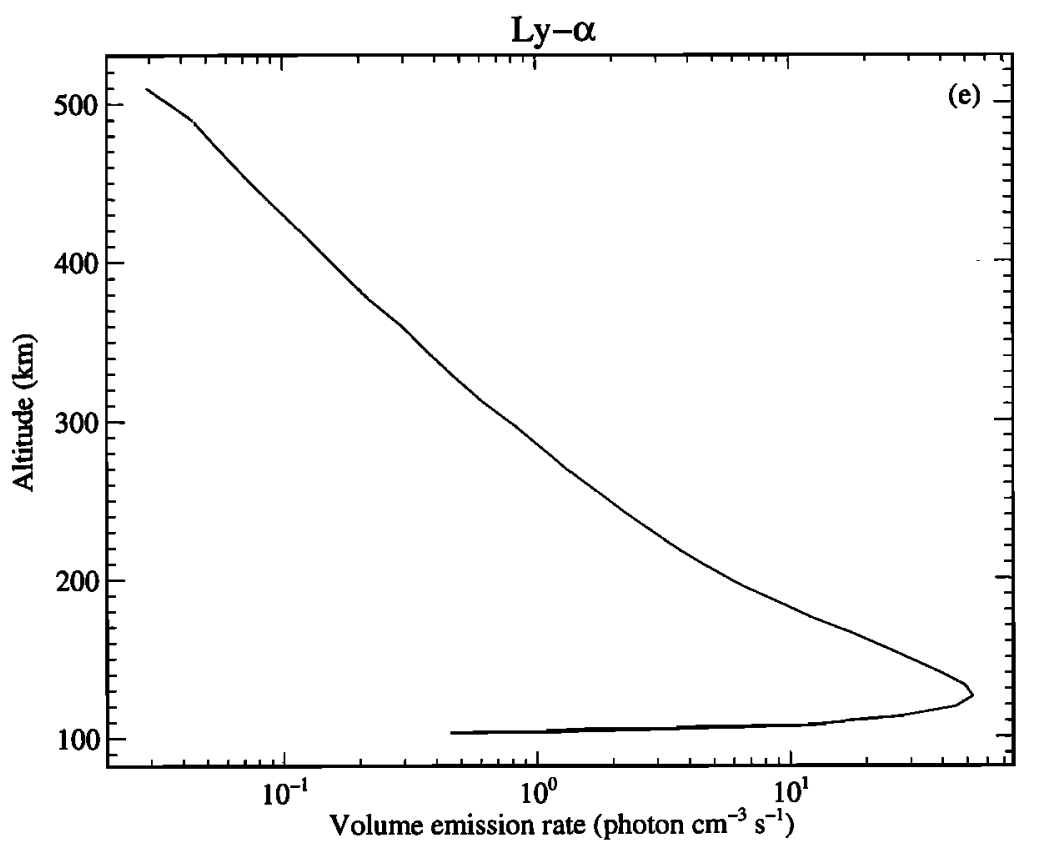

Figure 4. (continued)

Therefore direct electron impact is the second source of $1304 \AA$ excitation. Above $300 \mathrm{~km}$ the dayglow component (photoelectron impact and resonance scattering) becomes dominant. Figure 4d shows the N I $1493 \AA$ excitation sources. They closely follow the LBH-band excitation rate since the mechanism is identical and only threshold effects produce some differences. Finally, the emission rate of the Doppler-shifted Ly- $\alpha$ line (Figure 4e) shows a peak at the same altitude as that of the other emissions.

\section{Morphology of Proton-Induced FUV emission}

The three cases described in sections 3.2.1-3.2.3 clearly indicate that the relative role of electron and proton statistical precipitation in the excitation of the FUV emissions strongly varies with local time and latitude. In order to provide a global view of the role played by protons, we use Hardy et al.'s [1987, 1991] models to map the FUV emissions excited by both types of particles. We first establish the relationship between the FUV emerging emission rates for a nadir observation from a high-altitude orbit and the energy of the incoming protons and electrons. These curves (Figure 5) are obtained from the combined electron-proton code. A fixed neutral atmosphere corresponding to the protondominated aurora described before is used for all simulations.

Figure 5a shows the efficiency curve obtainied for the unabsorbed $\mathrm{N}_{2}$ LBH bands excited by electrons and protons. As was done previously, a Maxwellian distribution is assumed for the initial energy of the precipitated electrons. A fixed neutral atmosphere structure is used as it is not established that the MSIS model correctly reproduces the auroral composition in auroral conditions, especially during magnetically active periods. The sensitivity of these curves to the solar and geomagnetic conditions will be discussed in a later study. The electron efficiency reaches a maximum of $\sim 1.25$ LBH kR per incident erg $\mathrm{cm}^{-2} \mathrm{~s}^{-1}$ at $15 \mathrm{keV}$ and slowly decreases as a result of the increasing efficiency of the ionization channel at higher energies. At lower energies the LBH yield rapidly decreases, mainly owing to the drop of the $\mathrm{N}_{2}$ mixing ratio at higher altitudes where soft electrons deposit most of their energy. These values compare well with those derived by Strickland et al. [1993]. For example, for the $1700-1800 \AA$ region, a yield of $0.07 \mathrm{kR} / \mathrm{erg} \mathrm{cm} \mathrm{cm}^{-2} \mathrm{~s}^{-1}$ is calculated by Strickland et al. [1993] for electrons with a mean energy of $4 \mathrm{keV}$. Since this spectral band represents a fraction of 0.06 of the total LBH excitation, their total (unabsorbed) LBH yield is $1.2 \mathrm{kR} / \mathrm{erg} \mathrm{cm}^{-2} \mathrm{~s}^{-1}$, identical to our value for the same electron energy. At high energies, electrons penetrate deeper into the atmosphere where $\mathrm{O}_{2}$ absorption becomes significant (Figure $5 \mathrm{~b}$ ). This causes the efficiency to depart from the unabsorbed values and to drop to $0.2 \mathrm{kR} / \mathrm{erg} \mathrm{cm}^{-2} \mathrm{~s}^{-1}$ for $\bar{E}=50 \mathrm{keV}$.

To calculate the efficiency curve for protons, a kappa energy distribution is adopted with $\kappa=3.5$ for all energies. In the case of pure proton precipitation (Figure 5a) the unabsorbed LBH efficiency curve exhibits a behavior similar to that of the electrons. A peak value is reached following an increase of the nonabsorbed efficiency. The nearly equal values of the nonabsorbed efficiency for electrons and protons stem from the dominant role played by secondary electrons generated by the primary bean for both types of energetic particles. However, for a given energy, electrons penetrate deeper than protons do into the thermosphere [Rees, 1989; Solomon, 2001] as a consequence of the lower value of the total energy loss cross sections of electrons in the at- 

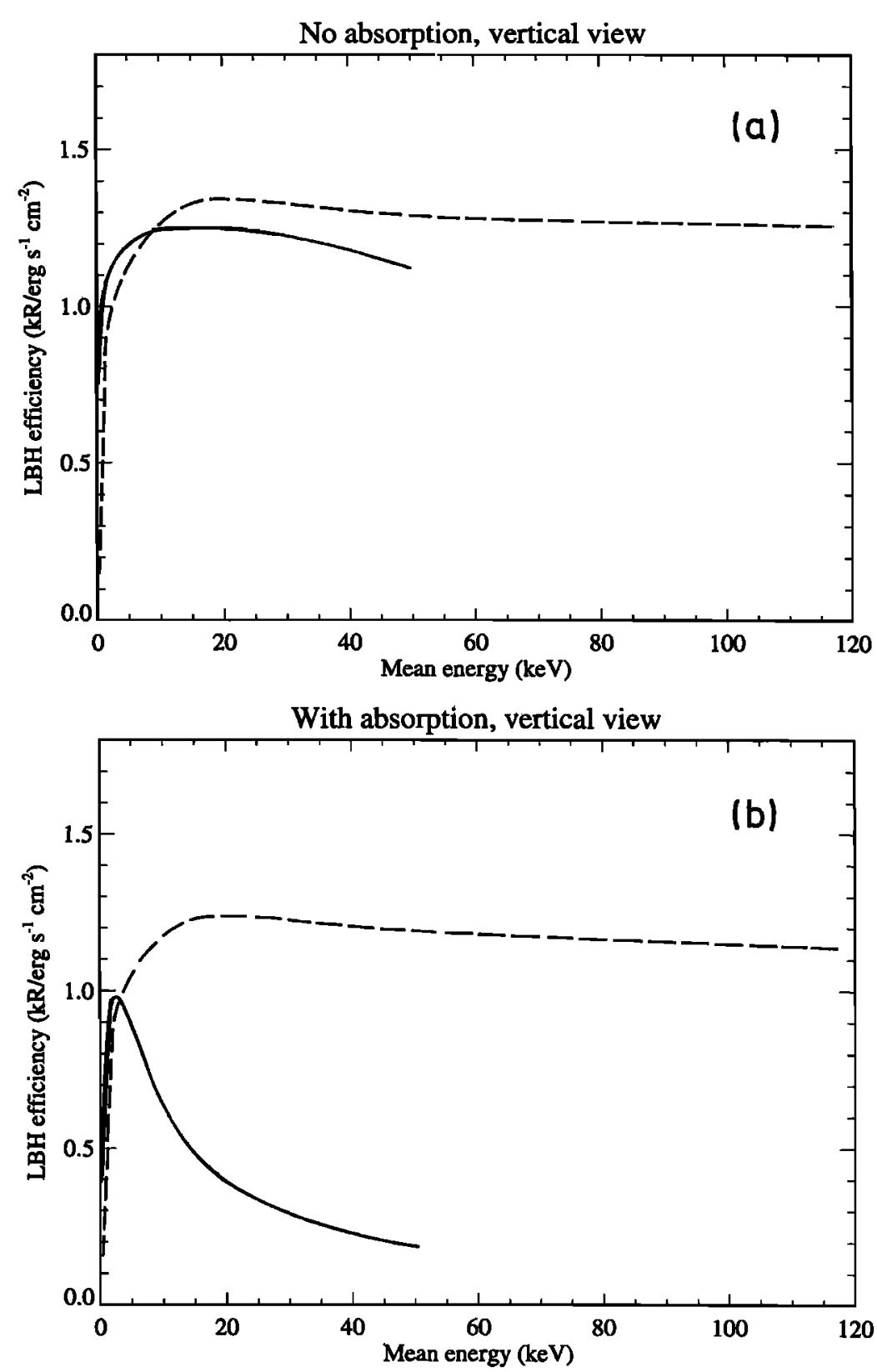

Figure 5. Efficiency of the emission rate of $\mathrm{N}_{2}$ LBH bands by electrons (solid line) and protons (dashed line) for a high-altitude nadir observation as a function of the mean particle energy: (a) no absorption, (b) with $\mathrm{O}_{2}$ absorption.

mospheric gases. The resulting difference in the altitude of the peak volume excitation rate for electrons and protons of a given energy produces a different absorption by $\mathrm{O}_{2}$, causing a lower efficiency of the electron precipitation case. The unabsorbed (Figure 5a) and absorbed (Figure 5b) proton curves are nearly identical up to $\sim$ $50 \mathrm{keV}$ since the bulk of the energy deposition occurs at altitudes above $120 \mathrm{~km}$ and is not significantly affected by $\mathrm{O}_{2}$ absorption. Our LBH efficiency values are close to those obtained by Strickland et al. [1993] at high energies. Their peak value for the $1325 \AA$ band is $60 \mathrm{R} / \mathrm{erg}$ $\mathrm{cm}^{-2} \mathrm{~s}^{-1}$, which corresponds to $1.2 \mathrm{kR} / \mathrm{erg} \mathrm{cm} \mathrm{cm}^{-2} \mathrm{~s}^{-1}$ to be compared with our maximum unabsorbed value of $1.35 \mathrm{kR} / \mathrm{erg} \mathrm{cm}^{-2} \mathrm{~s}^{-1}$. At lower energy, our curve drops to lower values than theirs does, possibly because of differences in the altitude of the energy deposition peak or the use of a different neutral composition.

Using the efficiency curves of Figure $5 \mathrm{~b}$ combined with the energy flux and mean energy of electrons and protons, the distribution of the total LBH emission rate in the (north) polar region for nadir observations may be calculated based on the basis of Hardy et al. 's [1985, 1991] models. Plates $1 \mathrm{a}$ and $1 \mathrm{~b}$ show the polar diagram (orthographic projections) of the nadir LBH brightness calculated for $K p=3$. In these plots the airglow component has not been included, as its brightness strongly depends on the solar illumination, that is on the day of the year, and solar activity. 

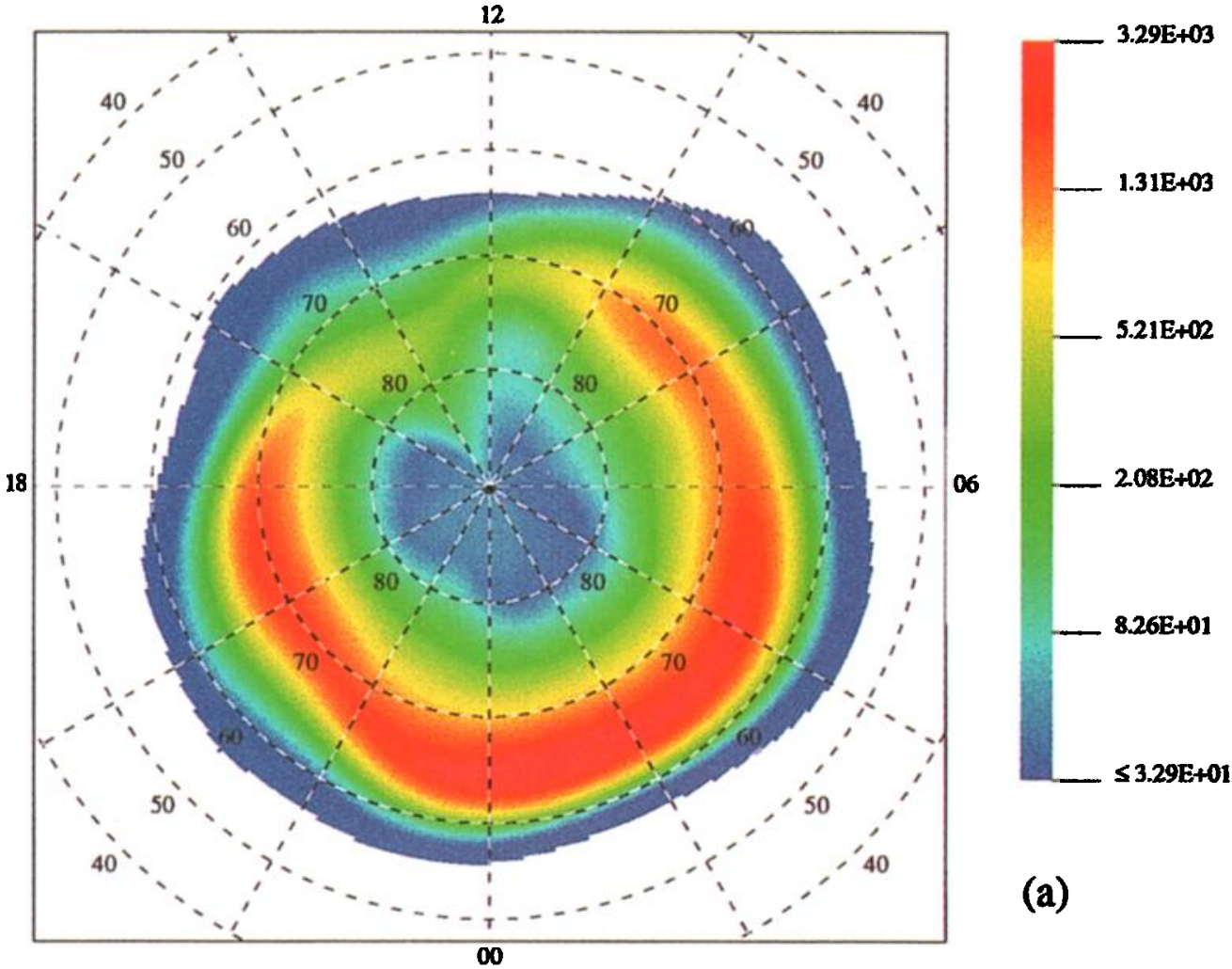

(a)
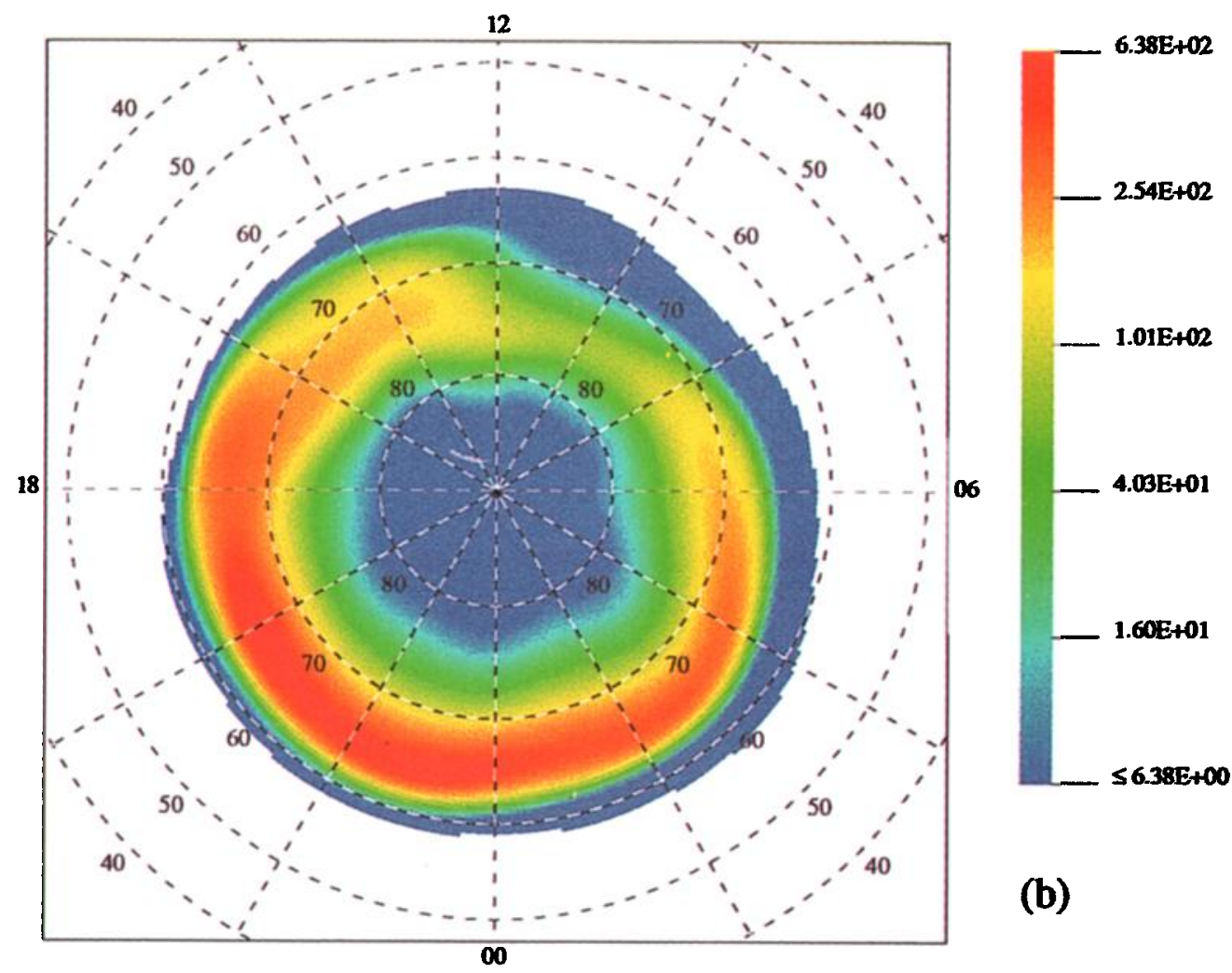

Plate 1. Polar plot (orthographic projection) of the statistical distribution of the calculated nadir $\mathrm{N}_{2}$ LBH emission (in Rayleighs) for $K p=3$ : (a) by electron; (b) by proton and (c) ratio of proton/electron excitation. 


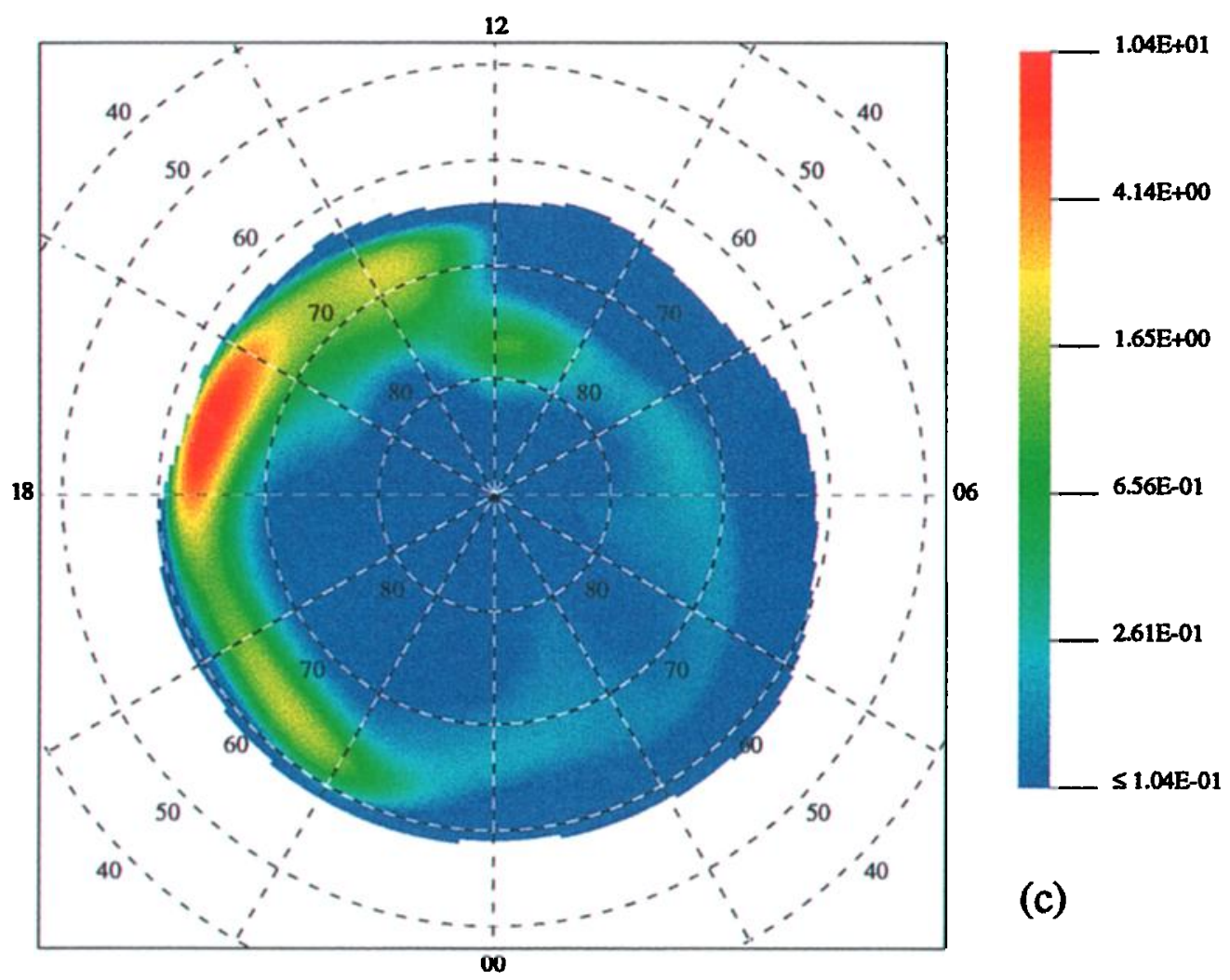

Plate 1. (continued) 


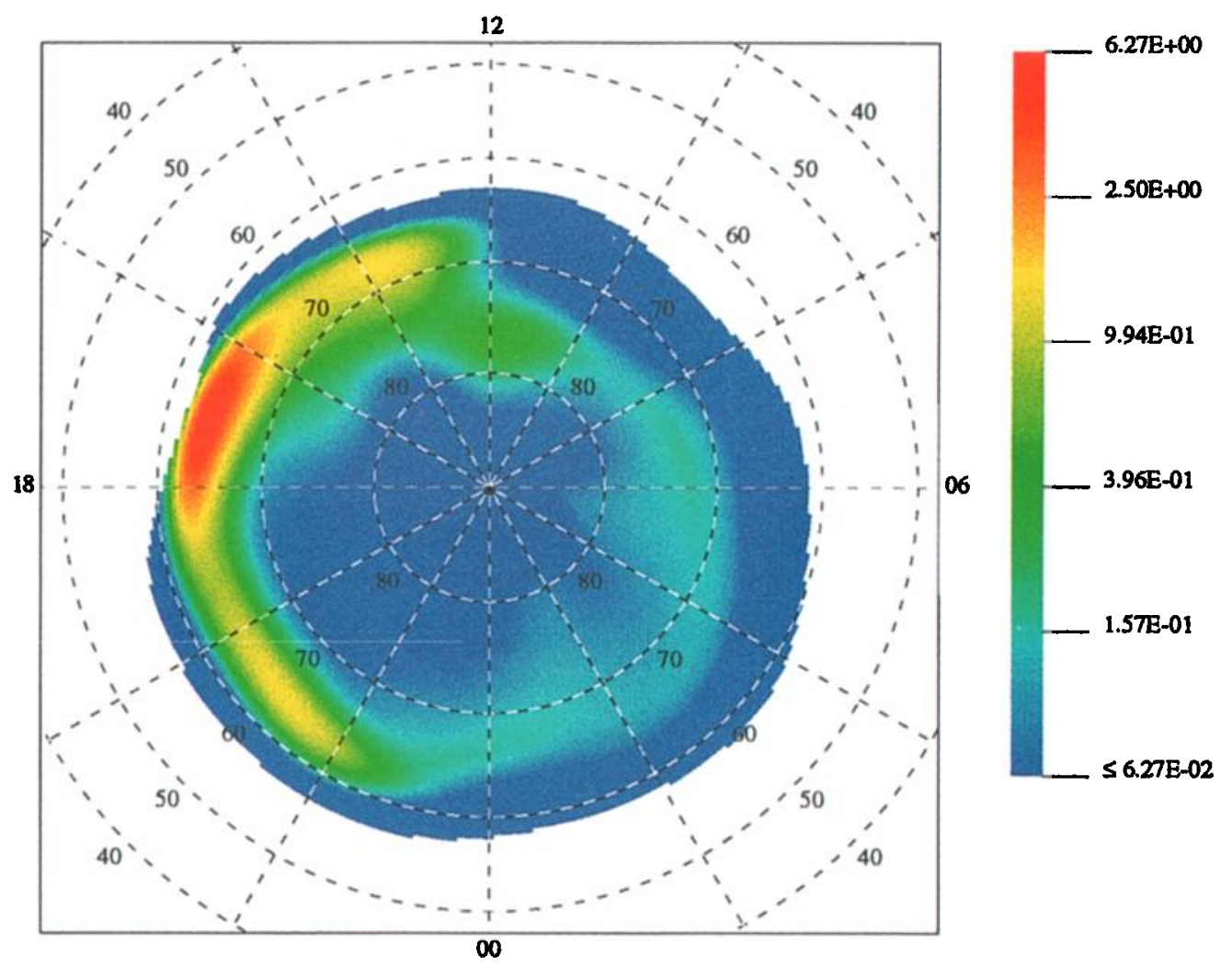

Plate 2. Polar plot of the distribution of the ratio of the proton to electron energy fluxes for $K p=3$.

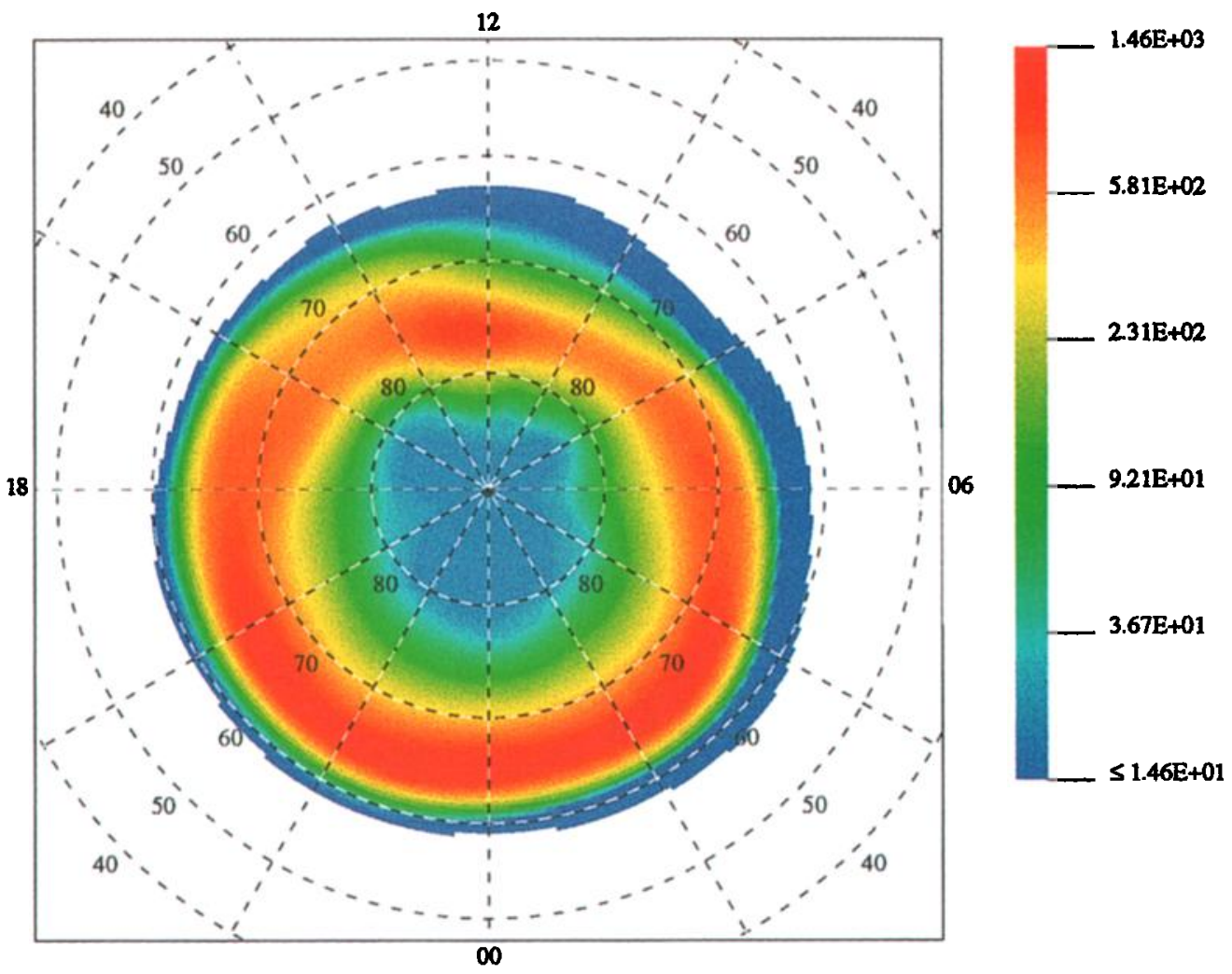

Plate 3. Polar plot of the distribution of the auroral Ly- $\alpha$ nadir emission rate (in Rayleighs) for $K p=3$. 

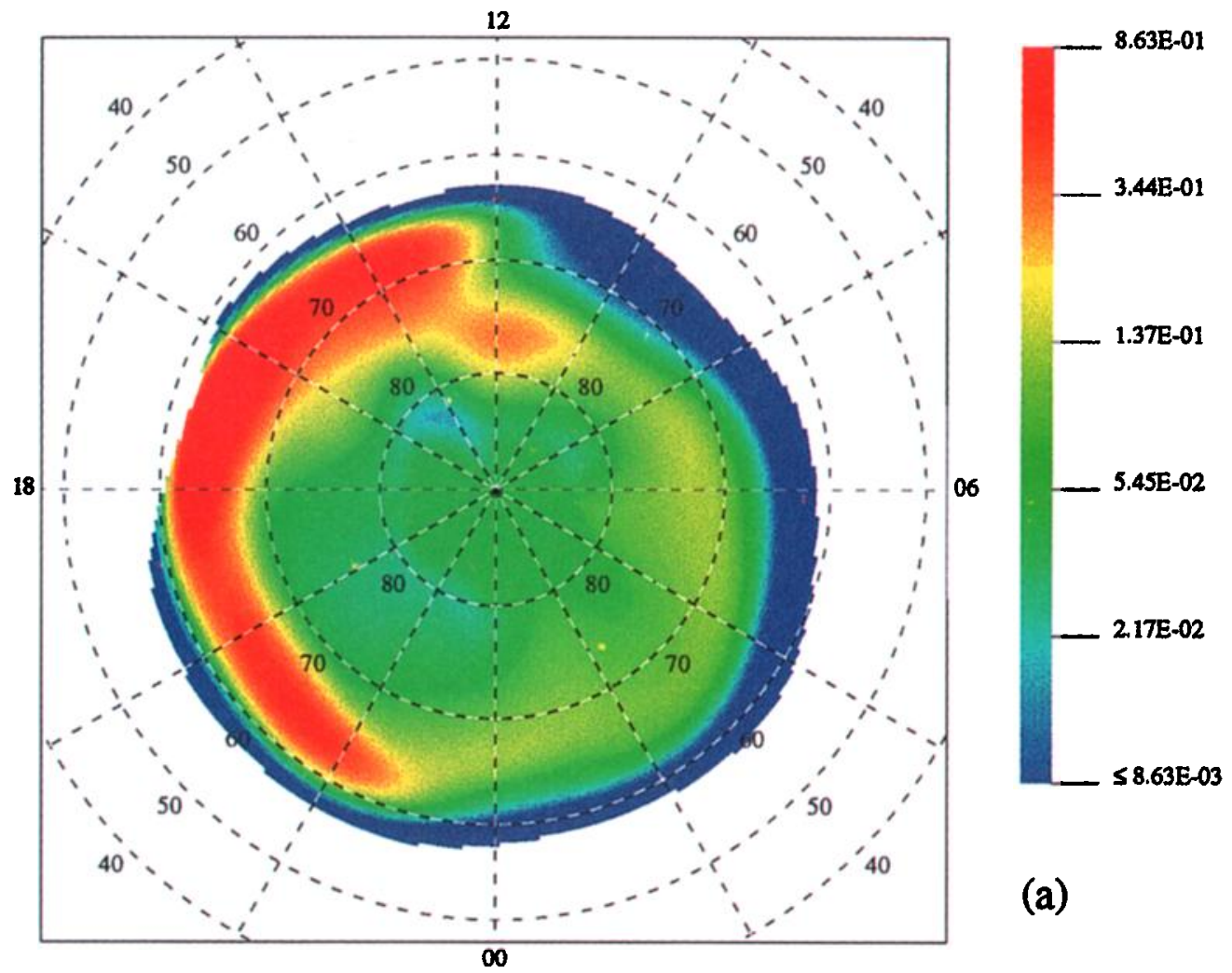

(a)
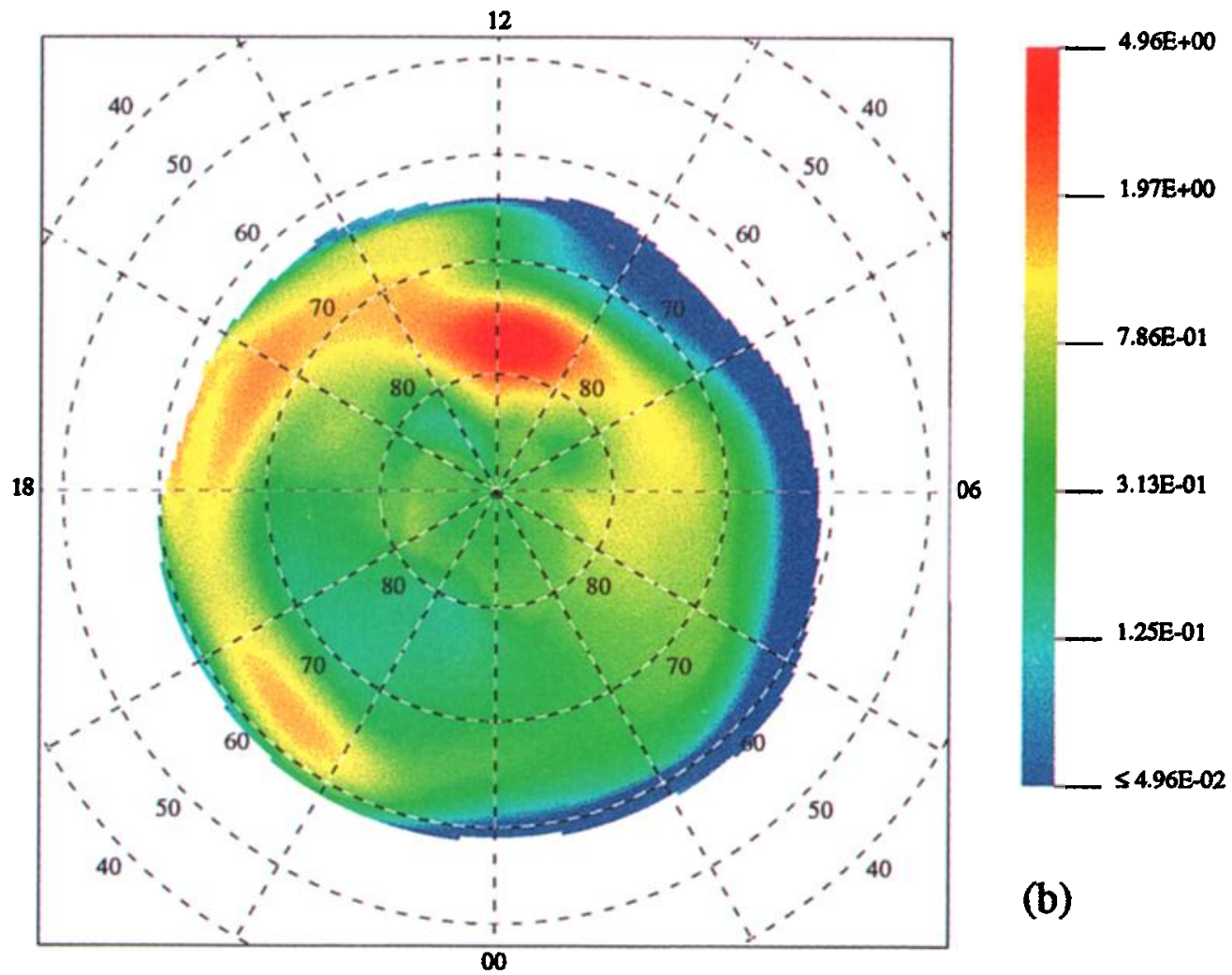

Plate 4. Validity of the Ly- $\alpha$ brightness as an indicator of the fraction of the energy flux precipitated by auroral protons: (a) ratio of the proton to the total (electron + proton) auroral energy flux and (b) intensity ratio of the Ly- $\alpha$ to the total (electron- and proton-excited) LBH nadir emission rates for $K p=3$. 
The electron-induced LBH distribution (Plate 1a) closely follows that of the total electron energy flux. The statistical mean electron energy varies from 0.1 to $10.5 \mathrm{keV}$ for $K p=3$, corresponding to emission peaks at $\sim 280$ and $\sim 105 \mathrm{~km}$, respectively, which causes limited absorption by $\mathrm{O}_{2}$. The largest value $\bar{E}=10.5 \mathrm{keV}$ is observed in the morning sector at $63^{\circ}$ in a region of weak electron precipitation and corresponds to a $\mathrm{LBH}$ column absorption of only $50 \%$. The efficiency conversion into LBH emission thus varies by a factor of 2 . In the daytime cusp sector where soft electrons with energies below $1 \mathrm{keV}$ are observed, the LBH efficiency drops to as low as $1 \mathrm{kR} / \mathrm{erg} \mathrm{cm}^{-2} \mathrm{~s}^{-1}$ at $1 \mathrm{keV}$ and $0.6 \mathrm{kR} / \mathrm{erg}$ $\mathrm{cm}^{-2} \mathrm{~s}^{-1}$ at $0.1 \mathrm{keV}$, as shown in Figure $5 \mathrm{~b}$.

A similar conclusion may be drawn for protons (Plate $1 b)$ where the $\mathrm{LBH}$ emission rate distribution resembles that of the proton energy flux. The mean proton energy ranges from $<0.1$ to $60 \mathrm{keV}$ (peak above 200 and at 125 $\mathrm{km}$, respectively), a range of energies unaffected by atmospheric absorption as shown before. A broad band of proton-induced LBH emission is calculated along a Cshaped region running from 1600 to 0500 MLT. We note that the drop of the proton excitation around 1000 MLT is more pronounced than the decrease of the proton energy flux is in this region. 'This difference is explained by the low energy of the precipitated protons.

Finally, the ratio of the proton-induced to electroninduced LBH intensity is shown in Plate 1c. It is seen that, globally, this map is quite similar to the distribution of the ratio of the proton/electron energy fluxes (Plate 2). Electron excitation is dominant relative to protons, especially in regions of high precipitated energy flux. However, protons make a nonnegligible contri- bution exceeding $10 \%$ over very broad regions, including sectors of strong precipitation. Regions of protondominated LBH excitation are observed near the equatorward boundary of the electron oval in the afternoon sector. The region of highest proton/electron excitation ratio runs from $68^{\circ}$ at $1300 \mathrm{MLT}$ to $63^{\circ}$ at 1900 MLT with a maximum value of 14 . A second, less pronounced maximum is obtained at $63^{\circ}$ between 2000 and 2200 MLT. Generally, regions of proton-dominated LBH excitation lie equatorward of the main electron precipitation in the afternoon and premidnight sectors. The largest proton/electron excitation ratio increases from 4.4 to 16.5 for $K p$ running from 0 to 5 . It remains located in the premidnight sector, equatorward of the main region of precipitation.

A region of visible aurora dominated by proton precipitation was also identified by Stenbaek-Nielsen et al. [1998] from a comparison between ground-based images and in situ particle measurements on board the Fast Auroral Snapshot Explorer (FAST) satellite during an overhead pass. It was located equatorward of the main electron-excited auroral arc in the premidnight sector. More generally, our results for the location of protondominated FUV emission are in agreement witl groundbased optical studies indicating the presence of diffuse proton auroral precipitation exceeding electrons in the premidnight sector.

As we did for the LBH bands, we calculate the variation of the $\mathrm{Ly}-\alpha$ vertical emission rate for various initial proton characteristic energies. The efficiency of the nadir emission rate for an incident $1 \mathrm{erg} \mathrm{cm}^{-2} \mathrm{~s}^{-1}$ proton precipitation is shown in Figure 6. When energy increases, the Ly- $\alpha$ efficiency decreases as ionization pro-

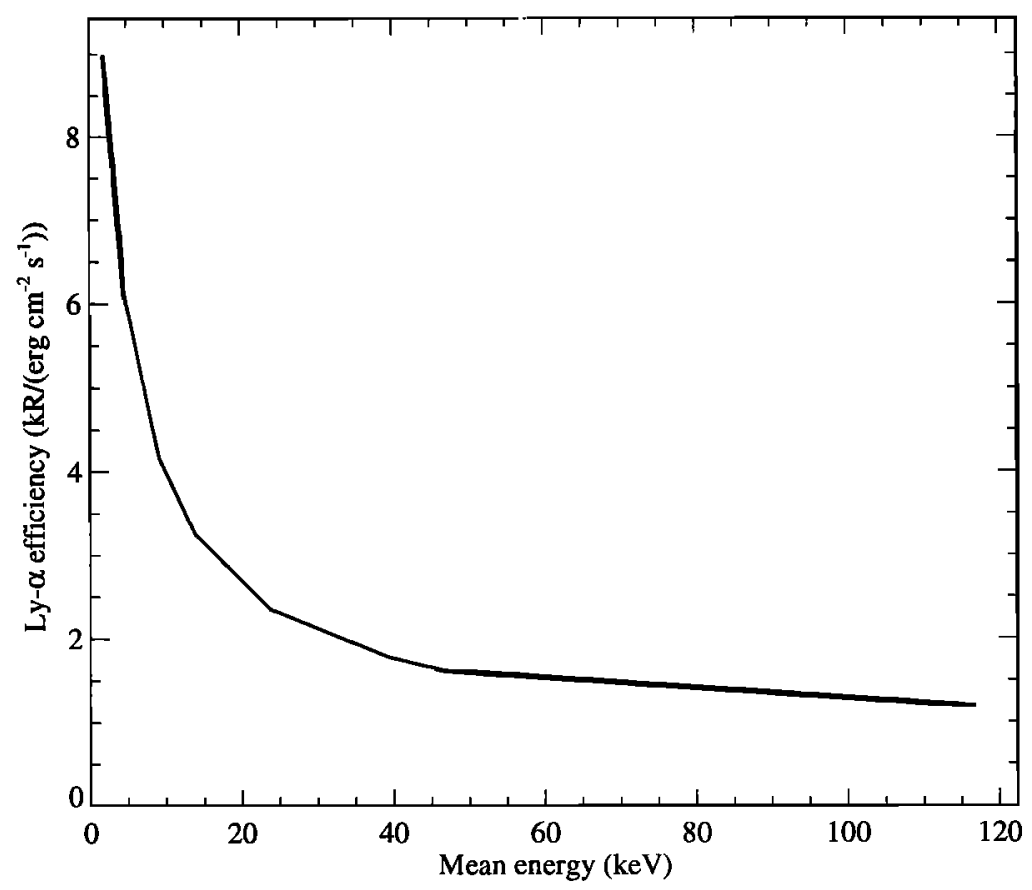

Figure 6. Efficiency of the Ly- $\alpha$ emission rate for a high-altitude nadir observation as a function of the mean proton energy. 
cesses (equation (1)) of major constituents are favored over charge exchange collisions.

For the range of mean proton energies encountered in the statistical precipitation model, no appreciable $\mathrm{O}_{2}$ absorption occurs. Consequently, the values in Figure 6 may also be considered as the emergent nadir efficiencies. Plate 3 shows the polar distribution of the Ly$\alpha$ brightness predicted by Hardy et al.'s [1989] model. This distribution is quite similar to that of the proton energy flux since $\mathrm{O}_{2}$ absorption is negligible, and the efficiency varies only by a factor of 2 over the characteristic proton energies encoutered in the statistical model. The maximum brightness is $1.5 \mathrm{kR}$, a value reached near midnight MLT at a latitude of $65^{\circ}$, that is, in a region of relatively strong electron precipitation. Locally and temporally, the proton energy flux may be larger than the statistical value used here. An example was described by Ishimoto et al. [1988] in a nighttime aurora for $K p=2$ where a Ly- $\alpha$ nadir intensity as high as $4 \mathrm{kP}$ was observed. This brightness exceeds that predicted using the statistical proton distribution. This difference strongly suggests that the actual fluxes encountered in a given spatial and temporal situation may be substantially larger than that given by Hardy et al.'s statistical models. Simulations for other values of $K p$ predict that the largest Ly- $\alpha$ nadir intensity in the polar regions steadily increases from $650 \mathrm{R}$ for $K p$ $=0$ to $2.2 \mathrm{kR}$ for $K p=5$.

Parallel remote sensing observation of the brightness of the Ly- $\alpha$ line and $\mathrm{N}_{2} \mathrm{LBH}$ bands may provide some estimate of the fraction of the total energy flux carried by protons and electrons. A comparison between the $\mathrm{Ly}-\alpha /$ total $\mathrm{LBH}$ ratio and the fraction of the total energy flux carried by auroral protons is illustrated in Plate 4. The $Q_{0, p} /\left(Q_{0, e}+Q_{0, p}\right)$ ratio measures the relative amount of energy associated with the proton precipitation (Plate $4 a$ ). The distribution of the Ly- $\alpha$ /total LBH ratio (Plate 4 b) shows similar features. A C-shaped region of maximum relative proton flux is observed from 1230 to $2300 \mathrm{MLT}$, with a higher latitude extension in the prenoon sector. The corresponding $\mathrm{Ly}-\alpha / \mathrm{LBH}$ ratio shows a similar C-shape although the relative minimum at 1900 MLT is less pronounced in the particle precipitation. The largest $\mathrm{Ly}-\alpha / \mathrm{LBH}$ ratio is predicted at $77^{\circ}$ near $1200 \mathrm{MLT}$, but the maximum proton/electron ratio is at $64^{\circ}$ and 1700 MLT. Comparison with Plate $1 \mathrm{~b}$ indicates, however, that both the proton flux and the Ly- $\alpha$ brightness are low in this sector. To determine a critical threshold where proton precipitation exceeds electrons, we calculated the $\mathrm{Ly}-\alpha / \mathrm{LBH}$ nadir intensity ratio associated with regions satisfying the condition $Q_{0, p}=Q_{0, e}$. For $K p=3$ we find that the associated $\mathrm{Ly}-\alpha / \mathrm{LBH}$ ratio is $1.1 \pm 0.2$. The dispersion is due to the different mean particle energies associated with any given value of the $Q_{0, p} /\left(Q_{0, e}+Q_{0, p}\right)$ ratio. The calculated $\mathrm{Ly}-\alpha / \mathrm{LBH}$ ratio varies from 0 (pure electron aurora) to 10 (pure $10-\mathrm{keV}$ proton aurora) as derived from Figure $5 \mathrm{~b}$. Consequently, a change of the observed ratio may, in principle, be interpreted in terms of variations in the proton or electron energy or in tlie fraction of energy carried by the protons. However, the similarity between Plates $4 \mathrm{a}$ and $4 \mathrm{~b}$ shows that flux variations are dominant with respect to efficiency changes. Nevertheless, the energy-precipitated flux ambiguity cannot be fully resolved without an independent determination of the proton energy.

\section{Summary and Conclusions}

An electron-proton transport model has been combined with energetic particle energy distributions derived from a statistical precipitation modei to calculate the emission rate of the mair features of the FUV auroral spectrum. Analysis of the various contributions indicates that except for Ly- $\alpha$, which is solely excited by protons and fast $\mathrm{H}$ atoms, electron excitation generally dominates over proton-induced emissions. In particular, for $K p=3$ the protons statistically contribute $23 \%$ of the LBH nadir intensity in the noon cusp and $17 \%$ in the midnight sector. Similarly, the proton-induced O I $1356 \AA$ emission contributes $6 \%$ in the noon cusp and $25 \%$ in the midnight sector. Generally, the LBH morphologies due to proton and electron excitation both resemble their respective statistical flux distributions. Statistical maps of the Ly- $\alpha$ intensity for high-altitude nadir observation show that the Ly- $\alpha$ brightness distribution approximately mimics that of the proton energy flux. The largest values are located in the midnight sector and reach $\sim 1.5 \mathrm{kR}$. The evening maximum is associated with the evening proton aurora identified by its ground-based optical signature in the $\mathrm{H} \mathrm{I}$ Balmer lines.

The region with the highest ratio of proton to electron precipitation is located equatorward of the main precipitation in the afternoon sector and is characterized by a $\sim 600 \mathrm{R} \mathrm{LBH}$ emission, $96 \%$ of which is produced by the proton precipitation component. Regions of FUV emission dominated by the proton component are predicted in the prenoon, afternoon, and evening sectors, equatorward of the main region of electron precipitation. Proton excitation is dominated by secondary electrons resulting from ionization of the major constituents by the energetic $\mathrm{H}^{+} / \mathrm{H}$ beam. Emission efficiency curves calculated for the LBH bands show that the effective yield for electron excitation significantly drops below $10 \mathrm{keV}$ owing to absorption by $\mathrm{O}_{2}$ of the shorter-wavelength $\mathrm{LBH}$ emission. Proton-induced LBH emission is unaffected by $\mathrm{O}_{2}$ absorption for the range of characteristic energies statistically encountered in the auroral proton precipitation.

Remote sensing of the Ly- $\alpha / \mathrm{LBH}$ ratio may be used to identify regions where the proton energy flux is a substantial fraction of the total auroral precipitated energy. Nonsunlit regions dominated by proton precipitation are characterized by a $\mathrm{Ly}-\alpha / \mathrm{LBH}$ ratio exceeding $\sim$ 1.1. As illustrated before, this ratio maps reasonably well the distribution of the relative proton flux, although it depends on both the mean energy of the particles and the reliative fraction of the proton flux. 
It should be noted that these conclusions are based on statistical particle energy distributions covering a wide range of seasons and solar activity levels. Models such as this one will be tiseful for analyzing FUV auroral emissions which will be observed on space missions such as IMAGE or Thermosphere Ionosphere Mesosphere Energetics and Dynamics (TIMED). A first comparison between simultaneous in situ particle measurements with FAS'T and global images obtained with IMAGE in the 1400-1700 $\AA$ passband [Frey et al., 2001] has been carried out using this model. It was shown that proton excitation of the LBH bands may be dominant near the equatorward edge of the quiet auroral oval, in good agreement with the results of this study.

Acknowledgments. J. C. G. is supported by the Belgian National Fund for Scientific Research (FNRS). This work was funded by the PRODEX program of the European Space Agency (ESA), the Belgian Fund for Collective Fundamental Research (grant FRFC 97-2.4569.97), and the Russian Foundation for Basic Research (RFBR grant 99-0217620).

Janet G. Luhmann thanks Dwight T. Decker and another referee for their assistance in evaluating this paper.

\section{References}

Ajello, J.M., and D.E. Shemansky, A reexamination of important $\mathrm{N}_{2}$ cross sections by electron impact with application to the dayglow: The Lyman-Birge-Hopfield band system and N I (119.99 nm), J. Geophys. Res., 90, 9845, 1985.

Banks, P.M., C.R. Chappell, and A.F. Nagy, A new model for the interaction of auroral electrons with the atmosphere: Spectral degradation, backscatter, optical emission and ionization, J. Geophys. Res., 79, 1459, 1974.

Basu, B., J.R. Jasperse, R.M. Robinson, R.R. Vondrak, and D.S. Evans, Linear transport theory of auroral proton precipitation: A comparison with observations, J. Geophys. Res., 92, 5920, 1987.

Basu, B., J.R. Jasperse, D.J. Strickland, and R.E. Daniell Jr., Transport-theoretic model for the electron-protonhydrogen atom aurora, J. Geophys. Res., 98, 21,517, 1993.

Bilitza, D. (Ed.), International Reference Ionosphere IRI 90, World Data Cent. for Rockets and Satellites, 90-22, Greenbelt, Md., 1990.

Christon, S.P., D.J. Williams, D.G. Mitchell, C.Y. Huang, and L.A. Frank, Spectral characteristics of plasma sheet ion and electron populations during disturbed geomagnetic conditions, J. Geophys. Res., 96, 1, 1991.

Conway, R. R., Multiple fluorescent scattering of $\mathrm{N}_{2}$ ultraviolet emissions in the atmospheres of the Earth and Titan, J. Geophys. Res., 88, 4784, 1983.

Creutzberg, F., R. L: Gattinger, F. R. Harris, S. Wozniak, and A. Vallance Jones, Auroral studies with a chain of meridian scanning photometers, 2, Mean distributions of proton and electron aurora as a function of magnetic activity, J. Geophys. Res., 93, 14,591, 1988.

Crosswhite, H.M., and W.G. Fastie, Far-ultraviolet auroral spectra, J. Opt. Soc. Am., 52, 643, 1962.

Decker, D.T., B.V. Kozelov, B. Basu, J.R. Jasperse, and V.E. Ivanov, Collisional degradation of the proton-H atom fluxes in the atmosphere: A comparison of theoretical techniques, J. Geophys. Res., 101, 26,947, 1996.

Eather, R.H., Auroral proton precipitation and hydrogen emissions, Rev. Geophys., 5, 207, 1967.
Edgar, B.C., W.T. Miles, and A.E.S. Green, Energy deposition of protons in molecular nitrogen and applications to proton auroral phenomena, J. Geophys. Res., 78, 6595, 1973.

Edgar, B.C., H.S. Porter, and A.E.S. Green, Proton energy deposition in molecular and atomic oxygen and applications to polar cap, Planet. Space Sci., 29, 787, 1975.

Feldman, P.D., and E.P. Gentieu, The ultraviolet spectrum of an aurora 530-1520 $\AA, J$. Geophys. Res., 87, 2453, 1982.

Frey, H.U., S.B. Mende, C.W. Carlson, J.-C. Gérard, B. Hubert, J. Spann, G.R. Gladstone, and T. J. Immel, The electron and proton aurora as seen by IMAGE-FUV and FAST, Geophys. Res. Lett. 28, 1135, 2001.

Galand, M., J. Lilensten, W. Kofman, and R.B. Sidje, Proton transport model in the ionosphere, 1, Multistream approach of the transport equations, J. Geophys. Res., 102, 22,261, 1997.

Gérard, J.C., and C.A. Barth, OGO-4 observations of the ultraviolet auroral spectrum, Planet. Space Sci., 24, 1059, 1976.

Gérard, J.C., B. Hubert, D.V. Bisikalo, and V.I. Shematovich, A model of the Lyman- $\alpha$ line profile in the proton aurora, J. Geophys. Res., 105, 15,795, 2000.

Germany, G.A., G.K. Parks, M. Brittnacher, J. Cumnock, D. Lummerzheim, J.F. Spann, L. Chen, P.G. Richards, and F.J. Rich, Remote determination of auroral energy characteristics during substorm activity, Geophys. Res. Lett, 24, 995, 1997.

Green, A.E.S., and R.S. Stolarski, Analytic models of electron impact excitation cross sections, J. Atmos. Terr. Phys., 34, 1703, 1972.

Hardy, D.A., M.S. Gussenhoven, and E. Holeman, A statistical model of auroral electron precipitation, J. Geophys. Res., 90, 4229, 1985.

Hardy, D.A., M.S. Gussenhoven, and R. Raistrick, Statistical and functional representations of the pattern of auroral energy flux, number flux, and conductivity, J. Geophys. Res., 92, 12,275, 1987.

Hardy, D.A., M.S. Gussenhoven, and D. Brautigam, A statistical model of auroral ion precipitation, J. Geophys. Res., 94, 370, 1989.

Hardy, D.A., W. McNeil, M.S. Gussenhoven, and D. Brautigam, A statistical model of auroral ion precipitation, 2, Functional representation of the average patterns, $J$. Geophys. Res., 96, 5539, 1991.

Hedin, A.E., Extension of the MSIS thermosphere model into the middle and lower atmosphere, J. Geophys. Res., 96, 1159, 1991.

Ishimoto, M., C.I. Meng, G.J. Romick, and R.E. Huffman, Auroral electron energy and flux from molecular nitrogen ultraviolet emissions observed by the S3-4 satellite, $J$. Geophys. Res., 93, 9854, 1988.

Ishimoto, M., C.I. Meng, G.R. Romick, and R.E. Huffman, Doppler shift of auroral Lyman $\alpha$ observed from a satellite, Geophys. Res. Lett., 16, 143, 1989.

Jackman, C.H., R.H. Garvey, and A.E.S. Green, Electron impact on atmospheric gases, 1, Updated cross sections, J. Geophys. Res., 82, 5081, 1977.

Jasperse, J.R., and B. Basu, Transport theoretic solutions for auroral proton and $H$ atom fluxes and related quantities, J. Geophys. Res., 87, 811, 1982.

Kozelov, B.V., and V.E. Ivanov, Monte-Carlo calculation of proton-hydrogen atom transport in $\mathrm{N}_{2}$, Planet. Space Sci., 40, 1503, 1992.

Lorentzen, D.A., F. Sigernes, and C.S. Deehr, Modeling and observations of dayside auroral hydrogen emission Doppler profiles, J. Geophys. Res., 103, 17,479, 1998.

Lummerzheim, D., and M. Galand, Profile of the $\mathrm{H}_{\beta}$ emission line in proton aurora, J. Geophys. Res., 106, 23, 2001. 
Lyons, L.R., and D.S. Evans, An association between discrete aurora and energetic particle boundaries, J. Geophys. Res., 89, 2395, 1984.

Marov, M. Y., V.I. Shematovich, D.V. Bisikalo, and J.C. Gérard, Nonequilibrium Processes in the Planetary and Cometary Atmospheres: Theory and Applications, Kluwer Acad., Norwell, Mass., 1997.

Mende, S.B., and R.H. Eather, Monochromatic all-sky observations and auroral precipitation patterns, J. Geophys. Res., 81, 3771, 1976.

Mende, S.B., et al., Far ultraviolet imaging from the IMAGE spacecraft, 1, System design, Space Sc. Rev., 91, 243, 2000.

Mende, S.B., H. Frey, S. B. Mende, H. U. Frey, M. Lampton, J.-C. Gérard, B. Hubert, S. Fuselier, R. Gladstone, and J. L. Burch, Global observations of proton and electron auroras in a substorm, Geophys. Res. Lett., 28, 1139, 2001.

Mumma, M.J., and E.C. Zipf, Dissociative excitation of vacuum-ultraviolet emission features by electron impact on molecular gases, II, $\mathrm{N}_{2}, J$. Chem. Phys., 55, 5582, 1971.

Ogawa, S., and M. Ogawa, Absorption cross sections of $\mathrm{O}_{2}\left(a^{1} \Delta_{g}\right)$ and $\mathrm{O}_{2}\left(\mathrm{X}^{3} \Sigma_{g}\right)$ in the region from 1087 to 1700 A, Can. J. Phys., 53, 1845, 1975.

Rees, M.H., On the interaction of auroral protons with the Earth's atmosphere, Planet. Space Scr., 30, 463, 1982.

Rees, M.H., Physics and Chemistry of the Upper Atmosphere, Cambridge Univ. Press, New York, 1989.

Robinson, R.M., R.R. Vondrak, K. Miller, T. Dabbs, and D. Hardy, On calculating ionospheric disturbances from the flux and energy of precipitating electrons, J. Geophys. Res., 92, 2565, 1987.

Rudd, M.E., Energy and angular distribution of secondary electrons from 5-100 keV proton collisions with hydrogen and nitrogen molecules, Phys. Rev. A, 20, 787, 1979.

Sharber, J.R., R.A. Frahm, J.D. Winningham, J.C. Biard, D. Lummerzheim, M.H. Rees, D.L. Chenette, E.E. Gaines, R.W. Nightingale, and W.L. Imhof, Observations of the UARS particle envirorirnent monitor and computation of ionzation rates in the middle and upper atmosphere during a geomagnetic storm, Geophys. Res. Lett., 20, 1319, 1993.
Solomon, S.C., Auroral particle transport using Monte Cário and Hybrid methods, J. Geophys. Res., 106, 107, 2001.

Solomon, S.C., P.B. Hays, and V. Abreu, The auroral $6300 \mathrm{~A}$ emission: Observation and modelling, J. Geophys. Res., 93, 9867, 1988.

Stenbaek-Nielsen, H.C., T.J. Hallinan, D.L. Osborne, J. Kimball, C. Chaston, J. McFadden, G. Delory, M. Temerin, and C.W. Carlson, Aircraft observations conjugate to FAST: Auroral arc thicknesses, Geophys. Res. Lett, 25, 2073, 1998.

Stone, E. J., and E. C. Zipf, Electron impact excitation on the ${ }^{3} S^{0}$ and ${ }^{5} S^{0}$ states of atomic oxygen, J. Chem. Phys., $60,4237,1974$.

Strickland, D.J., and D.E. Anderson, Radiation transport effects on the OI $1356-\AA$ limb intensity profile in the dayglow, J. Geophys. Res., 88, 9260, 1983.

Strickland, D.J., R.E. Daniell Jr., J.R. Jasperse, and B. Basu, Transport-theoretic model for the electron-protonhydrogen atom aurora, 2, Model results, J. Geophys. Res., 98, 21,533, 1993.

Synnes, S.A., F. Soraas, and J.P. Hansen, Monte Carlo simulations of proton aurora, J. Atmos. Sol. Terr. Phys., $60,1695,1998$.

Vallance-Jones, A., Aurora, D. Reidel, Norwell, Mass., 1974.

Van Zyl, B., and H. Neumann, Lyman $\alpha$ emission cross sections for low-energy $\mathrm{H}$ and $\mathrm{H}^{+}$collisions with $\mathrm{N}_{2}$ and $\mathrm{O}_{2}$, J. Geophys. Res., 93, 1023, 1988.

Zipf, E.C., and P. Erdman, Electron impact excitation of atomic oxygen: Revised cross section, J. Geophys. Res., 90, 11087, 1985.

B. Hubert and J.-C. Gérard, Laboratoire de Physique Atmosphérique et Planétaire, Institut d'Astrophysique et de Géophysique, 5 avenue de Cointe, B-4000 Liège, Belgium. (gerard@astro.ulg.ac.be; hubert@astro.ulg.ac.be)

Dimitri V. Bisikalo and Vladimir I. Shematovich, Institute of Astronomy, Russian Academy of Sciences, 48 Pjatnistkaja Street, Moscow, Russia. (bisikalo@inasan.rssi.ru; shematov@inasan.rssi.ru)

S. C. Solomon, Laboratory for Atmospheric and Space Physics, University of Colorado, Boulder, CO 80309. (solomon@lasp.colorado.edu)

(Received July 24, 2000; revised January 24, 2001; accepted January 31, 2001.) 